Research Article

\title{
Experimental and Numerical Research Studies on Multifield Coupling Rotordynamic Behaviors of Motor
}

\author{
Shaojie Guo $i$ and Changqing Bai $(D)$ \\ State Key Laboratory for Strength and Vibration of Mechanical Structures, \\ Shaanxi Key Laboratory of Environment and Control for Flight Vehicle, Xi'an Jiaotong University, Xi'an, 710049, China
}

Correspondence should be addressed to Changqing Bai; baichq@mail.xjtu.edu.cn

Received 2 March 2021; Accepted 7 June 2021; Published 2 July 2021

Academic Editor: Chao-Ping Zang

Copyright (C) 2021 Shaojie Guo and Changqing Bai. This is an open access article distributed under the Creative Commons Attribution License, which permits unrestricted use, distribution, and reproduction in any medium, provided the original work is properly cited.

\begin{abstract}
The magnetic pull, bearing forces, and operating temperature, as important factors affecting motor performance, actually act together on the electric motor. In this study, the magnetic-solid-thermal coupling rotordynamic behaviors of a three-phase asynchronous motor are investigated via experiments and numerical analysis. A motor test platform controlled by a variable frequency converter is offered, and the experiments under multiworking conditions are carried out to explore the rotordynamic characteristics of the motor system. A rotordynamic modeling method of the magnetic-solid-thermal coupling system is presented for analyzing the coupling effect of the unbalanced magnetic pull (UMP), nonlinear ball bearing forces (NBB), and operating temperature on the motor rotordynamic behaviors. All of the predicted results coincide well with the experimental data to validate the presented model. Through experiments and numerical analysis, it is shown that the interaction of magnetic, structural, and thermal fields plays a significant role in the nonlinear vibration of the motor rotor. UMP, even with slight amplitudes at low rotating speeds, can induce a remarkable impact on the dynamic characteristics of the system on account of the nonlinear effect of ball bearing forces. The combined action of UMP and NBB on the thermal effect is more notable than that of single action. The effect of the unbalanced load gradually decreases with the increase of the temperature. The experimental and numerical results indicate that the magnetic-solid-thermal coupling influence is a noticeable issue in the optimum design, failure diagnosis, and operation maintenance of motor systems.
\end{abstract}

\section{Introduction}

Electric motor is one of the essential and important components to realize the reciprocal transformation of electrical energy and mechanical energy. It is widely used in petroleum and chemical industry, automobiles, and aviation. With the increasing demand for higher motor performance, the accurate prediction of vibration characteristics of motor rotor systems is becoming more and more important in the design and operation. The motor rotor, which operates in the environment of multiphysical fields, suffers from the external forces including electromagnetic, mechanical, and aerodynamic forces, especially the first two actions. Simultaneously, the thermal effect, which has been the limiting factor for increasing the power limit of the motor, also has a significant impact on motor rotor systems.

The popularity of motors has led to an increasing interest in the dynamic performance of motors driven by the electromagnetic force acting on the stator and rotor. The difficulty of calculation and measurement of the unbalanced magnetic pull (UMP) acting on the rotor has been mentioned by Dorrell [1]. Transient measurements were carried out by Bradford [2] for accurate prediction of the magnitude of transient UMP during starting. Belmans et al. [3] analysed the relationship between the homopolar flux due to the eccentric rotor position and the UMP experimentally and theoretically. At recent decades, Guo et al. [4] used the numerical method to calculate the effect of relative 
eccentricity and pole number on the magnitude of the UMP. A rotordynamic simulation method was represented by Khalili et al. [5], and Kim and Nerg [6] included the effect of the UMP produced in a motor. In the abovementioned studies, the main attention is paid to the significant impact of UMP on the dynamic response of the motor rotor. But, some important factors affecting motor performance, such as the temperature rise and bearing, are not taken into account. It could lead to the vibration mechanism, and feature of motor rotors is difficult to be revealed effectively.

Otherwise, the ball bearing, which has been widely used in motor rotor systems for its low starting friction, high rigidity, and reliability, plays a key role in motor rotordynamic behaviors. The inherent nonlinearity of the ball bearings (NBB) has been widely and comprehensively investigated by many scholars [7-9]. Based on previous research studies, a general dynamic model was presented by Bai and $\mathrm{Xu}[10]$ for studying the nonlinear stability of a ball bearing-rotor system. Rafsanjani and Abbasion [11] analysed the nonlinear dynamic behavior of rolling element bearing systems considering the surface defects. In a recent study, the experimental and numerical study on the vibration response of an angular contact ball bearing with spall defect on the outer race was represented by Jafari and Rohani [12]. Unfortunately, only the impact of the ball bearing is considered in these works, while the UMP, which influences the rotordynamic behaviors of the motor, is neglected.

In above research studies, the significant effect of the heat on the action of UMP and NBB was not drawn into consideration, and the influences of the UMP and bearings were independently studied. As a result, the dynamic response of the motor rotor considering the combined effect of the electromagnetic, mechanical, and thermal factors could not be fully observed and interpreted. Till now, only a few of published articles had studied the nonlinear dynamic behavior under one or two of these factors. It is noted by Harris [13] that the friction in ball bearing could lead to the temperature rise, which could have an important impact on the rotor-bearing system. Later, Jeng and Huang [14] made an effort to improve the theoretical model proposed by Harris for predicting the temperature rise of the ball bearing. Recently, the results presented by Schmied [15], Lorenz and Murphy [16], Panara, et al. [17], Aydin et al. [18], and Plantegenet [19] showed that some symptoms of instabilities of the motor rotor are closely related to the thermal effect. Im et al. [20] found that the UMP was influenced by the variation of the air gap whose geometry would change accordingly with the temperature rise of the iron core. It was presented by Chuan and Gan [21] that the UMP was associated with the bearing friction. In [22], the numerical method was mainly employed by Guo and Bai for analyzing the effect of the combined action of UMP and NBB on the motor rotor system. However, only the magneticsolid coupling effect was considered in their work, and the thermal effect was not introduced in modeling and experiments. Up to now, numerical or/and experimental research studies on the coupling effect of electromagnetic, mechanical, and thermal factors are still scarce, especially the corresponding research studies combined with experiments.

Therefore, with respect to the above, the present study is intended to cast light on the nonlinear multifield coupling rotordynamic characteristics of a three-phase induction motor using experiments and numerical approach. A new 2DOF dynamic model of a motor rotor system is proposed under the coupling effect of the UMP, $\mathrm{NBB}$, and the temperature rise. A test rig of a motor whose rotor is supported by ball bearings is employed, and the experiments under multiworking conditions are carried out. Combined with a large number of experimental tests and numerical analysis based on the presented coupling dynamic model, the mechanism of magnetic-solid-thermal interaction and the influence of multifield coupling characteristics of the motor are investigated efficiently in this study.

\section{Experimental Investigation}

In order to investigate the dynamic characteristics of the motor rotor system under a multifield coupling condition, an experimental platform is employed, as shown in Figure 1. The motor whose rotating speed is controlled by SPWM (sinusoidal pulse width modulation) variable frequency drive (VFD) or inverter (MicroMaster 440) is mounted on the rigid base. The laser displacement sensor (M7L-4) with a resolution of $0.001 \mathrm{~mm}$ is placed vertically at the end of the shaft. The motor rotor is supported by two ball bearings at the two ends of the shaft. And a digital thermometer (TM-902C) is closely attached to the outer ring of the ball bearing. The displacement signals, obtained with the help of sensor, are input into a digital signal collector. And a data acquisition and processing system (DaqBook/2000A) is used to analyze the nonlinear dynamic behaviors of the motor rotor. The data acquisition and processing system utilizes a full period sampling as the data acquisition method. The specifications of the motor are given in Table 1, and the parameters of ball bearings (6205) are given in Table 2. The sampling rate of the data acquisition and processing system is $2049 \mathrm{~Hz}$, and the number of the sample data is 40,000. As shown in Figure 2, the value of output voltage increases with the rise of the supply frequency of VFD linearly. Dominated by VFD, the operating rotational speed of the motor rotor is strongly associated with the supply frequency in the test procedure. The signals of displacement and temperature rise of the rotor are recorded at different rotating speeds.

As given in Table 3, the experiment procedures can be categorized into two cases. Note that case 1 and case 2 are carried out to study the influence of rotating speed and temperature rise on the motor rotor dynamic response, respectively. In case 1, the constant temperature is set for the test when the rotating speed of the motor rotor varies from $597 \mathrm{rpm}$ to $2695 \mathrm{rpm}$. The motor needs to be naturally cooled to the corresponding room temperature $\left(23^{\circ} \mathrm{C}\right)$ during each operation to ensure the same operating temperature of the motor at each speed. On the other hand, in the experiment of temperature variation from $30^{\circ} \mathrm{C}$ to $50^{\circ} \mathrm{C}$ 


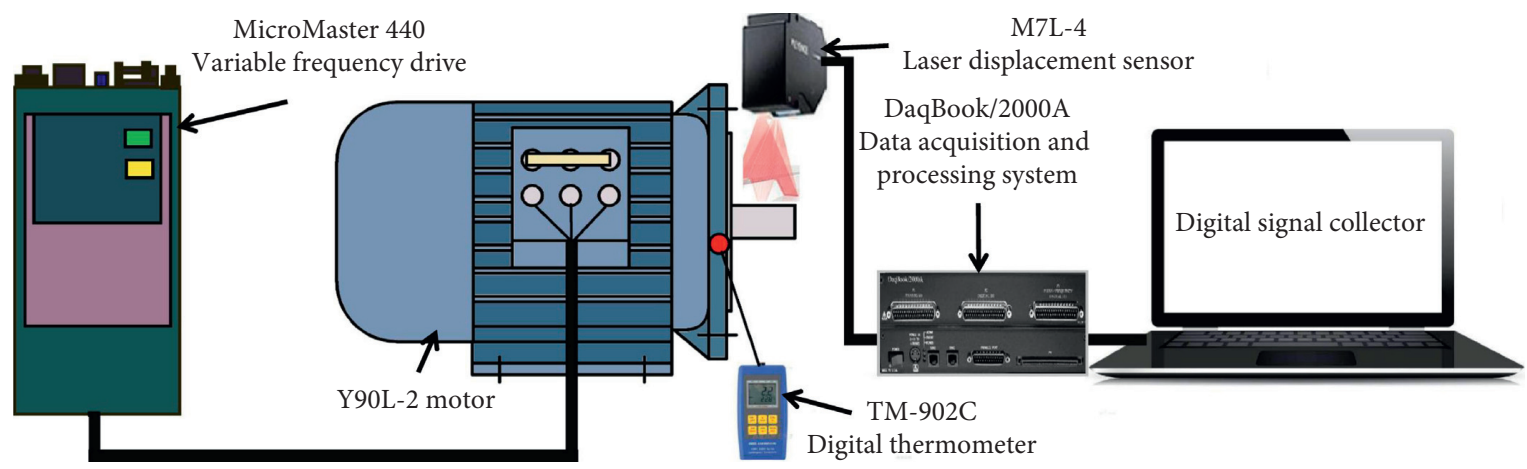

FIGURE 1: Experiment assembly of the motor test system.

TABle 1: Specification of the motor.

\begin{tabular}{lc}
\hline Parameter & Value \\
\hline Rated power & $2.2 \mathrm{~kW}$ \\
Rated voltage & $220 \mathrm{~V}$ \\
Number of pole pairs $(p)$ & 1 \\
Number of stator slots $\left(Z_{1}\right)$ & 18 \\
Number of rotor slots $\left(Z_{2}\right)$ & 16 \\
Core length $(L)$ & $110 \mathrm{~mm}$ \\
Outer radius of rotor $\left(R_{10}\right)$ & $35.65 \mathrm{~mm}$ \\
Average air gap length $\left(\delta_{0}\right)$ & $0.35 \mathrm{~mm}$ \\
Rotor mass $(m)$ & $3.49 \mathrm{~kg}$ \\
\hline
\end{tabular}

TABLE 2: Specification of ball bearing.

\begin{tabular}{lc}
\hline Bearing parameter & Value \\
\hline Number of balls $(N)$ & 9 \\
Ball diameter $\left(D_{b 0}\right)$ & $7.938 \mathrm{~mm}$ \\
Pitch diameter $D_{p}$ & $38.5 \mathrm{~mm}$ \\
Inner race curvature radius $\left(r_{i}\right)$ & $4.09 \mathrm{~mm}$ \\
Outer race curvature radius $\left(r_{o}\right)$ & $4.17 \mathrm{~mm}$ \\
Inner race clearance $\left(c_{i}\right)$ & $0.0025 \mathrm{~mm}$ \\
Outer race clearance $\left(c_{o}\right)$ & $0.0025 \mathrm{~mm}$ \\
\hline
\end{tabular}

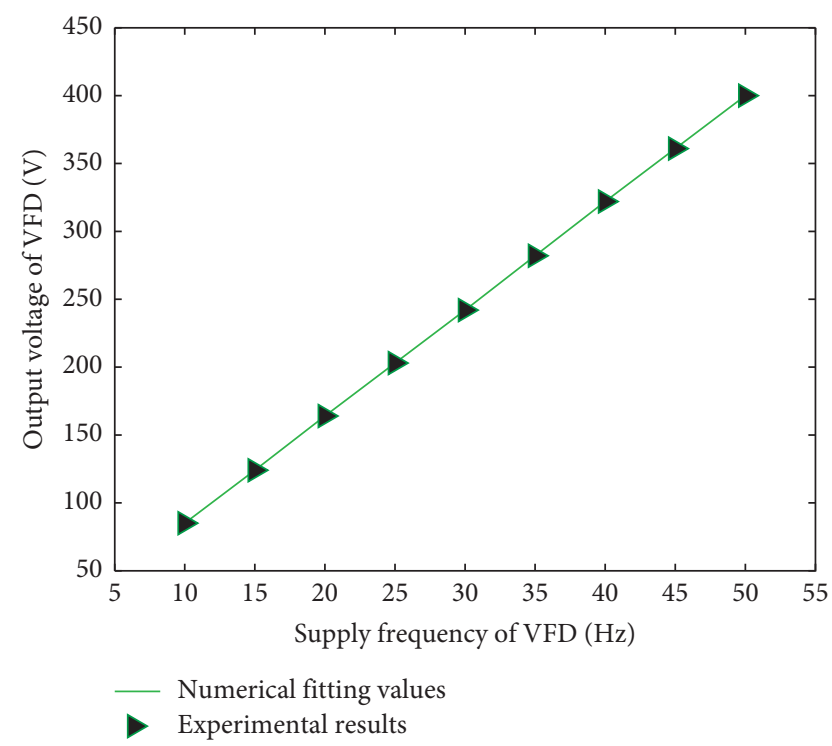

FIGURE 2: Amplitude variation of output voltage versus supply frequency of VFD. (case 2), the rotating speed of the motor rotor is kept at $1196 \mathrm{rpm}$.

\section{Nonlinear Multifield Coupling Rotordynamic Modeling}

The sketch map of a three-phase induction motor is shown in Figure 3. A coordinate system is defined for numerical analysis, where $X, Y$ are the radial coordinate directions, and $Z$ is the axial coordinate direction of the rotor. The motor mainly consists of a fixed stator and a rotor with two ball bearings supporting both ends. In order to study the dynamic behavior of the motor rotor under the multifield coupling condition, the UMP generated by the magnetic field as well as the nonlinear bearing forces under the specific temperature should be determined first, and then, the differential equations of motion for the system can be formulated.

3.1. Thermal Effect on the Motor Rotor System. The loss generated during the operation of the motor eventually turns into thermal energy, which leads to the temperature rise of the motor. The thermal resistance between the rotor and the stator is much larger than those of other parts due to the existence of air gap and axial airflow. Thus, the heat exchange between the rotor and the stator can be neglected in the temperature rise prediction for the motor [23].

As shown in Figure 4, the main losses of the motor rotor stem from the iron core and the copper windings of the rotor. The heat generated by the copper windings is transmitted to the surrounding environment through three ways. Part of the heat is transferred from the end of the windings to the air, and the corresponding thermal resistance is $R_{C 1}$. Some of the heat is transferred to the air from the surface of the windings near the duct, and the corresponding thermal resistance is $R_{C 2}$. The rest of heat is passed to the core and then from the core to the air. At the same time, the heat produced by loss in the iron core can be transmitted to the environment through four parts: the ducts $R_{F 1}$, the inner surface of the iron core $R_{F 2}$, the outer surface of the iron core $R_{F 3}$, and the copper windings $R_{C F}$. The thermal resistance between the copper windings and iron core is denoted as $R_{C F}$. According to the heat circuit diagram 
TABle 3: Specification of experiment procedures.

\begin{tabular}{lccc}
\hline Case & Supply frequency of VFD $(\mathrm{Hz})$ & Rotating speed $(\mathrm{rpm})$ & Temperature $\left({ }^{\circ} \mathrm{C}\right)$ \\
\hline & 10 & 597 & 897 \\
& 15 & 1196 & 1497 \\
& 20 & 1795 & 23.00 \\
Case 1 & 25 & 2096 & 2394 \\
& 30 & 2695 & 31.55 \\
& 35 & & 34.25 \\
& 40 & & 36.00 \\
& 45 & & 38.68 \\
Case 2 & & & 41.95 \\
& & & 46.17 \\
& & & 47.50 \\
& & & 50.04 \\
\hline
\end{tabular}

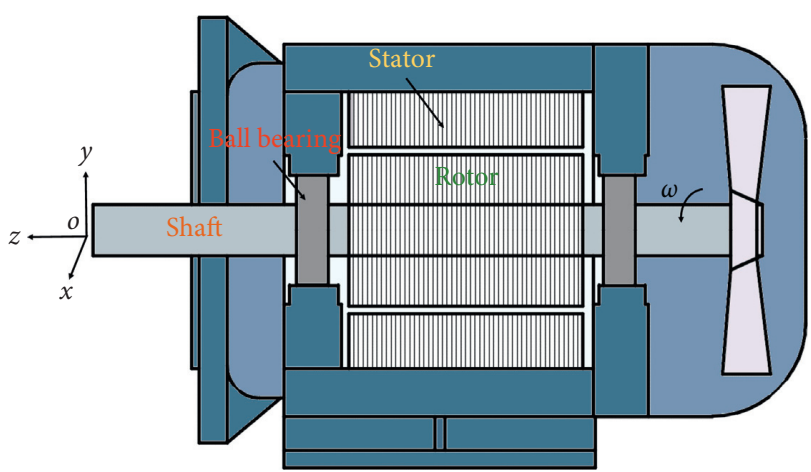

FIGURE 3: A description of a three-phase induction motor.

of two heat sources shown in Figure 4, the following equations can be listed:

$$
\left\{\begin{array}{l}
\left(\frac{\Delta T_{C}-\Delta T_{F}}{R_{C F}}\right)+\frac{\Delta T_{C}}{R_{\mathrm{Cu}}}=p_{C} \\
\left(\frac{\Delta T_{C}-\Delta T_{F}}{R_{C F}}\right)+p_{F}=\frac{\Delta T_{F}}{R_{\mathrm{Fe}}}
\end{array}\right.
$$

where $\Delta T_{C}$ and $\Delta T_{F}$ are the temperature rise of the copper windings and the rotor iron core, respectively. $p_{F}$ and $p_{C}$ are the power losses of the iron core and copper windings, respectively. $R_{\mathrm{Fe}}$ is the total thermal resistance of iron core, which is given as $1 / R_{\mathrm{Fe}}=\left(1 / R_{F 1}+1 / R_{F 2}+1 / R_{F 3}\right) \cdot R_{\mathrm{Cu}}$ is the total thermal resistance of rotor windings, which can be obtained from $1 / R_{\mathrm{Cu}}=\left(1 / R_{\mathrm{C} 1}+1 / R_{\mathrm{C} 2}\right)$.

Otherwise, the friction in a ball bearing as in most other mechanisms represents a wasteful power dissipation manifested in the form of heat generation [24]. The heat will finally cause the temperature of the bearings to rise. The bearing frictional power loss related to the rotating speed $n$ and the ball bearing torque $M$ can be expressed as $H_{A}=1.047 \times 10^{-4} \mathrm{nM}$. Note that $M$ consists of four types of torque such as viscous friction torque, torque due to applied load, spinning force, and gyroscopic force acting on the

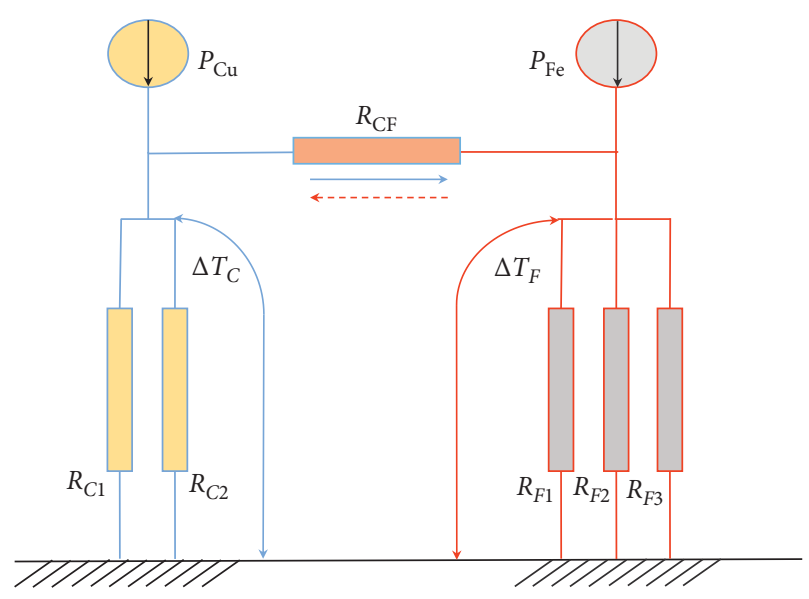

FIgURE 4: The thermal circuit diagram of the motor rotor.

bearing balls. The detailed expressions of the thermal resistances and the bearing torques can be obtained from Appendix. The relevant variables are listed in Nomenclature.

The dominant part of the heat generated by the ball bearing described by Harris was dissipated via heat convection. Therefore, the effects of heat conduction and radiation are neglected in this study. The heat convection between the ball bearing surface and atmosphere, $H_{1}$, and the convection between the balls and the compressed air flow, $H_{2}$, occur simultaneously. According to [14], $H_{1}$ can be expressed as follows:

$$
H_{1}=h_{v 1} \cdot S_{1} \cdot \Delta T_{B},
$$

where $S_{1}$ is the external area of a bearing housing. $\Delta T_{B}$ is the temperature rise of the ball bearing. $h_{v 1}$ is the outside film coefficient of heat transfer. $H_{2}$ can be given as

$$
H_{2}=h_{v 2} \cdot S_{2} \cdot \Delta T_{B} \text {, }
$$

where $S_{2}$ is the total area of the surface of the bearing balls. $h_{v 2}$ is the coefficient of heat transfer for air.

The heat balance equation can be established based on the assumption that the heat generated by the ball bearing, 
$H_{A}$, is mainly dissipated via heat convection, which can be described as $H_{A}=H_{1}+H_{2}$. Then, the temperature rise of the ball bearing can be determined according to the heat balance equation.

3.2. Nonlinear Ball Bearing Force. The geometry of a ball bearing in a frame of axes $o, x, y, z$ is shown in Figure 5. During the rotor operation, the temperature change of the outer ring of the bearing is more insignificant than that of the rotating parts. Therefore, the volume variation of the housing due to the heat influence can be ignored. Relatively, the thermal effect has a remarkable impact on the temperature rise of bearing balls, which could lead to the change of bearing clearance via the expansion of ball volume. According to the thermal effect analysis represented in Section 3.1, the diameter of the balls, $D_{b}$, can be expressed as

$$
D_{b}=D_{b 0}\left(\bar{\lambda}_{2} \cdot \Delta T_{B}+1\right),
$$

where $\bar{\lambda}_{2}$ is the thermal expand coefficient of the bearing balls. $D_{b 0}$ is the diameter of the bearing balls at room temperature.

When the outer race is stationary and the inner race is displaced under the bearing loading, the elastic deformation of the rolling element can be expressed as

$$
\delta_{j}=\left(x \cos \varphi_{j}+y \sin \varphi_{j}\right) \cos \alpha-\left[c_{o}+c_{i}-D_{b 0}\left(\bar{\lambda}_{2} \cdot \Delta T_{B}\right)\right],
$$

where $c_{o}$ and $c_{i}$ are the internal radial clearances between the inner and outer races. $\alpha$ is the contact angle which is related to the clearance. $\varphi_{j}$ is the angular location of the $j^{\text {th }}$ rolling element, which can be obtained from

$$
\varphi_{j}=\frac{2 \pi(j-1)}{N}+\omega_{c} t+\varphi_{0},
$$

where $N, t$, and $\varphi_{0}$ are the number of balls, time, and the initial angular location, respectively. The angular velocity of the cage $\omega_{e}$ can be expressed as

$$
\omega_{c}=\frac{1}{2} \omega\left(1-\frac{D_{b 0}\left(\bar{\lambda}_{2} \cdot \Delta T_{B}+1\right)}{D_{p}} \cos \alpha\right),
$$

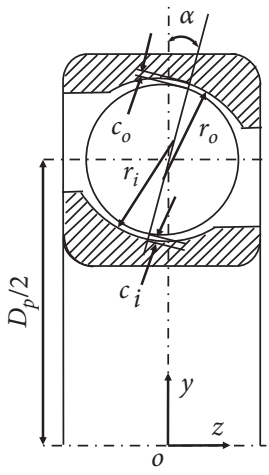

(a)

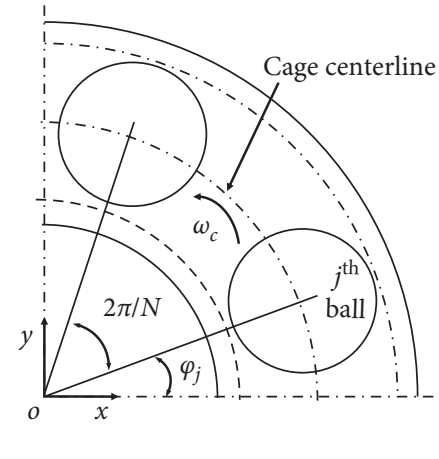

(b)
FIGURE 5: Geometry in ball bearing.

where $\omega$ is the angular velocity of the motor rotor, and $D_{\mathrm{b} 0}$ and $D_{p}$ are the ball diameter and bearing pitch diameter, respectively. To simplify the analysis, $\omega^{*}$ is defined as the rotating frequency of the rotor. $\omega^{*}$ can be expressed as $\omega^{*}=\omega /(2 \pi)$, where the superscript "*" denotes the unit conversion of the corresponding parameter from radian to the frequency. This superscript definition is also applied in the following content. When $\omega_{c}^{*}$ is the rotating frequency of the cage, $\omega_{c}^{*}=\omega_{c} /(2 \pi)$; the varying compliance frequency or the ball passage frequency $\omega_{\mathrm{vc}}^{*}$ can be described as $\omega_{\mathrm{vc}}^{*}=\omega_{\mathrm{vc}} /(2 \pi)$.

When the contact deformation $\delta_{j}$ is positive, the contact force could be calculated through the Hertzian contact theory; otherwise, no load is transmitted. So the contact force can be obtained by the following equation [13]:

$$
Q_{j}= \begin{cases}K_{j} \delta_{j}^{3 / 2}, & \delta_{j}>0, \\ 0, & \delta_{j} \leq 0,\end{cases}
$$

where $K_{j}$ and $\delta_{j}$ are the contact stiffness and the contact deformation of the $j^{\text {th }}$ ball, respectively. It is noted that the Hertzian contact force of bearings could be impacted by the bearing clearance associated with the temperature rise.

Thus, the total bearing force $F^{B}$ along $X$ and $Y$ directions can be expressed by summing the contact forces for each rolling element as follows:

$$
\left\{\begin{array}{l}
F_{x}^{B}=\sum_{j=1}^{N} \gamma_{j} K_{j}\left\{\left(x \cos \varphi_{j}+y \sin \varphi_{j}\right) \cos \alpha-\left[c_{o}+c_{i}-D_{b 0}\left(\bar{\lambda}_{2} \cdot \Delta T_{B}\right)\right]\right\}^{3 / 2} \cos \varphi_{j} \cos \alpha \\
F_{y}^{B}=\sum_{j=1}^{N} \gamma_{j} K_{j}\left\{\left(x \cos \varphi_{j}+y \sin \varphi_{j}\right) \cos \alpha-\left[c_{o}+c_{i}-D_{b 0}\left(\bar{\lambda}_{2} \cdot \Delta T_{B}\right)\right]\right\}^{3 / 2} \sin \varphi_{j} \cos \alpha
\end{array}\right.
$$

where $\gamma_{j}$ is a coefficient with respect to the relationship between the contact deformation $\delta_{j}$ and the ball bearing force:

$$
\gamma_{j}= \begin{cases}1, & \delta_{j}>0, \\ 0, & \delta_{j} \leq 0 .\end{cases}
$$


3.3. Unbalanced Magnetic Pull. The air gap of a motor with an eccentric rotor is shown in Figure 6. When the motor is operating, the temperature variation of the stator is less significant than that of the rotor. Therefore, the size change of the stator can be neglected. Otherwise, the thermal effect could influence the temperature of the rotor, which can result in the change of the air gap through the thermal expansion of the rotor iron core. The temperature rise of the rotor iron core can be obtained by solving (1). The outer radius of the rotor under the specific temperature can be expressed as

$$
R_{1}=R_{10}\left(\bar{\lambda}_{1} \cdot \Delta T_{F}+1\right),
$$

where $R_{10}$ is the rotor outer radius under the normal condition of room temperature, and $\bar{\lambda}_{1}$ is thermal expansion coefficient of the rotor core.

The air gap, $\delta$, between the stator and rotor can be approximately expressed as follows:

$$
\delta(\theta, t)=\left[R_{2}-R_{10}\left(\bar{\lambda}_{1} \cdot \Delta T_{F}+1\right)\right]-\delta_{e} \cos (\theta-\omega t),
$$

where $\theta$ is the value of the angular coordinate, $t$ is the time, and $\omega$ is the angular velocity of the motor rotor. The transient displacement of the rotor center, $\delta_{e}$, could be calculated by $\delta_{e}=\sqrt{\left(x_{t}^{2}+y_{t}^{2}\right)}$. Note that $x_{t}$ and $y_{t}$ are the displacements of the rotor along $X$ and $Y$ directions.

According to [25], the UMP generated by the air gap magnetic field is defined as

$$
\sigma=\frac{B^{2}(\theta, t)}{2 \mu_{0}},
$$

where $\mu_{0}$ is the air permeance. $B^{2}(\theta, t)$ is the magnetic flux density distribution of the air gap magnetic field, which is given as [4]

$$
B(\theta, t)=F(\theta, t) \Lambda(\theta, t),
$$

where $F(\theta, t)$ and $\Lambda(\theta, t)$ are the fundamental magnetomotive force (MMF) of the air gap and the magnetic permeance per unit of the air gap, respectively. In the threephase motor under no load, $F(\theta, t)$ can be derived from the following equation.

$$
F(\theta, t)=F_{j} \cos \left(\omega_{s} t-p \theta\right),
$$

where $\omega_{s}$ and $p$ are the angular velocity of power and the pole pair number of the motor, respectively. The amplitude of the fundamental MMF of the excitation current of the rotor, $F_{j}$, can be estimated by $F_{j}=N I$, where $N$ is the number of turns of windings, and $I$ is the effective current.

The magnetic permeance per unit of the air gap is expanded by Guo et al. [4] as a Fourier series as

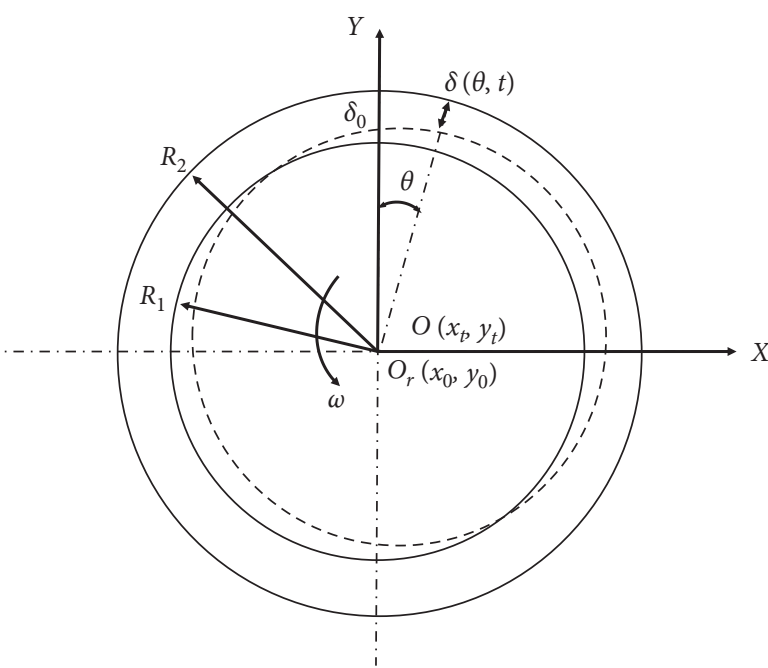

FIgURe 6: Air gap of a motor with an eccentric rotor.

$$
\begin{aligned}
\Lambda(\theta, t) & =\sum_{n=0}^{\infty} \varepsilon^{n} \cos ^{n}(\theta-\omega t) \\
& =\sum_{n=0}^{\infty} \Lambda^{n} \cos [n(\theta-\omega t)], \\
\Lambda_{n} & = \begin{cases}\frac{\mu_{0}}{\delta_{0}} \frac{1}{\sqrt{1-\varepsilon^{2}}}, & (n=0), \\
\frac{2 \mu_{0}}{\delta_{0}} \frac{1}{\sqrt{1-\varepsilon^{2}}}\left(\frac{1-\sqrt{1-\varepsilon^{2}}}{\varepsilon}\right)^{n}, & (n>0),\end{cases}
\end{aligned}
$$

where $\varepsilon$ is the relative eccentricity, which can be expressed as $\varepsilon=\delta_{e} / \delta_{0} . \delta_{0}$ is the mean air gap distance, which is given as $\delta_{0}=\left[R_{2}-R_{10}\left(\bar{\lambda}_{1} \cdot \Delta T_{F}+1\right)\right]$. It is noted that the length of air gap, which is related to the temperature rise, can have an impact on the electromagnetic force.

Thus, the Maxwell stress over rotor surface $\sigma$ can be obtained from (16). The UMP along $X$ and $Y$ directions can be calculated by integrating the horizontal and vertical components of the Maxwell stress $\sigma$ along the circumference of the rotor.

$$
\left\{\begin{array}{l}
F_{x}^{E}=R_{10}\left(\bar{\lambda}_{1} \cdot \Delta T_{F}+1\right) L \int_{0}^{2 \pi} \sigma(\theta, t) \cos (\theta) \mathrm{d} \theta, \\
F_{y}^{E}=R_{10}\left(\bar{\lambda}_{1} \cdot \Delta T_{F}+1\right) L \int_{0}^{2 \pi} \sigma(\theta, t) \sin (\theta) \mathrm{d} \theta,
\end{array}\right.
$$

where $R_{1}$ and $L$ are the rotor outer radius and the length of the rotor core, respectively.

Substituting (15) and (16) into (14), the first four terms of the infinite series yield can be substituted into (13). In the case of the resulting force, UMP can be expressed as 


$$
\left\{\begin{array}{l}
F_{x}^{E}=\frac{R_{10}\left(\bar{\lambda}_{1} \cdot \Delta T_{F}+1\right) L \pi}{4 \mu_{0}} F_{j}^{2}\left(2 \Lambda_{0} \Lambda_{1}+\Lambda_{1} \Lambda_{2}+\Lambda_{2} \Lambda_{3}\right) \cos (\omega t) \\
+\frac{R_{10}\left(\bar{\lambda}_{1} \cdot \Delta T_{F}+1\right) L \pi}{4 \mu_{0}} F_{j}^{2}\left(\Lambda_{0} \Lambda_{1}+\frac{1}{2} \Lambda_{1} \Lambda_{2}+\frac{1}{2} \Lambda_{2} \Lambda_{3}\right) \cos \left(2 \omega_{s} t-\omega t\right) \\
+\frac{R_{10}\left(\bar{\lambda}_{1} \cdot \Delta T_{F}+1\right) L \pi}{4 \mu_{0}} F_{j}^{2}\left(\Lambda_{0} \Lambda_{3}+\frac{1}{2} \Lambda_{1} \Lambda_{2}\right) \cos \left(2 \omega_{s} t-3 \omega t\right), \\
F_{y}^{E}=\frac{R_{10}\left(\bar{\lambda}_{1} \cdot \Delta T_{F}+1\right) L \pi}{4 \mu_{0}} F_{j}^{2}\left(2 \Lambda_{0} \Lambda_{1}+\Lambda_{1} \Lambda_{2}+\Lambda_{2} \Lambda_{3}\right) \sin (\omega t) \\
+\frac{R_{10}\left(\bar{\lambda}_{1} \cdot \Delta T_{F}+1\right) L \pi}{4 \mu_{0}} F_{j}^{2}\left(\Lambda_{0} \Lambda_{1}+\frac{1}{2} \Lambda_{1} \Lambda_{2}+\frac{1}{2} \Lambda_{2} \Lambda_{3}\right) \sin \left(2 \omega_{s} t-\omega t\right) \\
-\frac{R_{10}\left(\bar{\lambda}_{1} \cdot \Delta T_{F}+1\right) L \pi}{4 \mu_{0}} F_{j}^{2}\left(\Lambda_{0} \Lambda_{3}+\frac{1}{2} \Lambda_{1} \Lambda_{2}\right) \sin \left(2 \omega_{s} t-3 \omega t\right) .
\end{array}\right.
$$

3.4. Two Degrees of Freedom Equations of Motion of the Rotor System. In our study, the motor rotor is horizontal and symmetrical, which is approximated as a Jeffcott rotor. It is assumed that the shaft is rigid enough that its deformation can be ignored, so the radial displacement of the rotor center can be regarded as consistent with that of the bearing center.
The constant mass eccentricity, which is concentrated on the axis center of the rotor, should be taken into account, as well as the rotor gravity $W$. Thus, the 2 DOF equation of motion of the ball bearing-rotor system considering the UMP, the ball bearing effects, and the thermal effects can be expressed as 


$$
\begin{aligned}
& \int m \frac{\partial^{2} x}{\partial t^{2}}+c \frac{\partial x}{\partial t}+\sum_{j=1}^{N} \gamma_{j} K_{j}\left\{\left(x \cos \varphi_{j}+y \sin \varphi_{j}\right) \cos \alpha-\left[c_{o}+c_{i}-D_{b 0}\left(\bar{\lambda}_{2} \cdot \Delta T_{B}\right)\right]\right\}^{3 / 2} \cos \varphi_{j} \cos \alpha \\
& =\frac{R_{10}\left(\bar{\lambda}_{1} \cdot \Delta T_{F}+1\right) L \pi}{4 \mu_{0}} F_{j}^{2}\left(2 \Lambda_{0} \Lambda_{1}+\Lambda_{1} \Lambda_{2}+\Lambda_{2} \Lambda_{3}\right) \cos (\omega t) \\
& +\frac{R_{10}\left(\bar{\lambda}_{1} \cdot \Delta T_{F}+1\right) L \pi}{4 \mu_{0}} F_{j}^{2}\left(\Lambda_{0} \Lambda_{1}+\frac{1}{2} \Lambda_{1} \Lambda_{2}+\frac{1}{2} \Lambda_{2} \Lambda_{3}\right) \cos \left(2 \omega_{s} t-\omega t\right) \\
& +\frac{R_{10}\left(\bar{\lambda}_{1} \cdot \Delta T_{F}+1\right) L \pi}{4 \mu_{0}} F_{j}^{2}\left(\Lambda_{0} \Lambda_{3}+\frac{1}{2} \Lambda_{1} \Lambda_{2}\right) \cos \left(2 \omega_{s} t-3 \omega t\right)+m e \omega^{2} \sin (\omega t), \\
& m \frac{\partial^{2} y}{\partial t^{2}}+c \frac{\partial y}{\partial t}+\sum_{j=1}^{N} \gamma_{j} K_{j}\left\{\left(x \cos \varphi_{j}+y \sin \varphi_{j}\right) \cos \alpha-\left[c_{o}+c_{i}-D_{b 0}\left(\bar{\lambda}_{2} \cdot \Delta T_{B}\right)\right]\right\}^{3 / 2} \sin \varphi_{j} \cos \alpha \\
& =-W+\frac{R_{10}\left(\bar{\lambda}_{1} \cdot \Delta T_{F}+1\right) L \pi}{4 \mu_{0}} F_{j}^{2}\left(2 \Lambda_{0} \Lambda_{1}+\Lambda_{1} \Lambda_{2}+\Lambda_{2} \Lambda_{3}\right) \sin (\omega t) \\
& +\frac{R_{10}\left(\bar{\lambda}_{1} \cdot \Delta T_{F}+1\right) L \pi}{4 \mu_{0}} F_{j}^{2}\left(\Lambda_{0} \Lambda_{1}+\frac{1}{2} \Lambda_{1} \Lambda_{2}+\frac{1}{2} \Lambda_{2} \Lambda_{3}\right) \sin \left(2 \omega_{s} t-\omega t\right) \\
& -\frac{R_{10}\left(\bar{\lambda}_{1} \cdot \Delta T_{F}+1\right) L \pi}{4 \mu_{0}} F_{j}^{2}\left(\Lambda_{0} \Lambda_{3}+\frac{1}{2} \Lambda_{1} \Lambda_{2}\right) \sin \left(2 \omega_{s} t-3 \omega t\right)+m e \omega^{2} \cos (\omega t)
\end{aligned}
$$

where $m, c$, and $e$ are the total mass of the rotor, the damping coefficient of the ball bearings, and the mass eccentricity of the rotor, respectively. $x$ and $y$ are the radial displacements of rotor center along $X$ and $Y$ directions. The last components of the external loads in equation (20) is the unbalanced force due to the rotor mass eccentricity, which is time-varying and related to $e, m$, and $\omega$.

In this study, the Newmark- $\beta$ method is employed to solve the equation of motion of the ball bearing-rotor system. Equation (20) can be converted to the equations of motion of the rotor system without the UMP easily, which is

$$
\left\{\begin{array}{l}
m\left(\frac{\partial^{2} x}{\partial t^{2}}+c \frac{\partial x}{\partial t}\right)+\sum_{j=1}^{N} \gamma_{j} K_{j}\left\{\left(x \cos \varphi_{j}+y \sin \varphi_{j}\right) \cos \alpha-\left[c_{o}+c_{i}-D_{b 0}\left(\bar{\lambda}_{2} \cdot \Delta T_{B}\right)\right]\right\}^{3 / 2} \cos \varphi_{j} \cos \alpha=m e \omega^{2} \sin (\omega t), \\
m\left(\frac{\partial^{2} y}{\partial t^{2}}+c \frac{\partial y}{\partial t}\right)+\sum_{j=1}^{N} \gamma_{j} K_{j}\left\{\left(x \cos \varphi_{j}+y \sin \varphi_{j}\right) \cos \alpha-\left[c_{o}+c_{i}-D_{b 0}\left(\bar{\lambda}_{2} \cdot \Delta T_{B}\right)\right]\right\}^{3 / 2} \sin \varphi_{j} \cos \alpha=-W+m e \omega^{2} \cos (\omega t) .
\end{array}\right.
$$

It is noted that the nonlinear force of ball bearings shown in equations (9) and (21) should be replaced by $F_{x}^{B}=K^{*} x$, $F_{y}^{B}=K^{*} y$, and $F_{y}^{B}=K^{*} y$ when the nonlinearity of ball bearings (NBB) is not involved. $K^{*}$ could be approximated as the equivalent linear stiffness of ball bearings.

\section{Nonlinear Rotordynamic Behavior Analysis of the Motor}

4.1. The Influence of the Rotor Rotating Speed. In order to investigate the influence of the rotating speed on dynamic 
response of the motor rotor, numerical and experimental methods are employed to obtain the rotor dynamic behavior versus rotating speeds at room temperature. Figures 7-8 show the comparison between the results of numerical calculations and experiments. As shown in Figure 7(a), the vertical displacement signals are acquired at different times, along with the increased rotating speed. The related frequency spectrum is shown in Figure 7(b). It can be found that the predicted results are in good agreement with experimental data, thus validating the proposed model. The rotating speed of the motor rotor is mainly controlled by the frequency of the output voltage of VFD. Thus, in Table 4, when the output power frequency of VFD is set to $10 \mathrm{~Hz}$, $15 \mathrm{~Hz}, 20 \mathrm{~Hz}, 25 \mathrm{~Hz}, 30 \mathrm{~Hz}, 35 \mathrm{~Hz}, 40 \mathrm{~Hz}$, or $45 \mathrm{~Hz}$, the corresponding rotating speed of the motor rotor is $597 \mathrm{rpm}$, $897 \mathrm{rpm}, 1196 \mathrm{rpm}, 1497 \mathrm{rpm}, 1795 \mathrm{rpm}, 2096 \mathrm{rpm}$, $2394 \mathrm{rpm}$, and $2695 \mathrm{rpm}$. In the comparison of the frequency spectrum shown in Figure 7(b), we selected the frequency range from zero to five times the supply frequency, in which there are mainly five frequency components $\left(\omega^{*}, 2 \omega^{*}\right.$, $3 \omega^{*}, 4 \omega^{*}$, and $\left.5 \omega^{*}\right)$. Among them, the fundamental frequency $\omega^{*}$, which is stemmed from the combined action of rotor mass eccentricity and the UMP, has the highest value. Obtained by numerical results, the frequency components of $3 \omega^{*}, 4 \omega^{*}$, and $5 \omega^{*}$ with the lower amplitudes are also consistent with those of the test data. Their impact on the rotor dynamic response is much smaller than $\omega^{*}$ and $2 \omega^{*}$. Figure 8 shows the amplitude of $\omega^{*}$ and $2 \omega^{*}$ versus the rotating speed of the motor rotor at the same room temperature. The amplitude deviation between the experimental and numerical results is within $6 \%$. The curve of $\omega^{*}$ has a significant nonlinearity, which is the result of the coupling effect of UMP and NBB. Due to the influence of aerodynamic force and other external factors, the amplitude curve of $\omega^{*}$ obtained from the experiment is slightly higher than that of the numerical results. Compared to $\omega^{*}$, the amplitude of the amplitude curve of $2 \omega^{*}$ is less sensitive to changes of the rotating speed, but its impact on the dynamic behavior of the rotor should not be neglected.

In order to distinguish the impacts of the UMP and the $\mathrm{NBB}$ on the rotor dynamic response at the same temperature, three different cases are taken into consideration: UMP only, NBB only, and both of the UMP and NBB. By solving the two degrees of freedom equations of motion of the motor rotor system, we are able to obtain the dynamic response of the bearing-rotor system under different cases.

Figure 9(a) illustrates the rotor orbits of the presented dynamic model under different cases, and the maximum amplitude in $Y$ direction versus rotating speed is shown in Figure 9(b). For further analysis, the maximum amplitudes of forces in $Y$ direction versus rotating speed under different cases are shown in Figure 10. When only the UMP is considered, the rotor orbit, as shown in Figure 9(a) (black line), presents as a circle. Its radius gradually rises with the increasing rotating speed of the rotor, resulting in the nonlinear growth of maximum displacement in $Y$ direction as shown in Figure 9(b). This phenomenon is caused by the increase of the amplitude of the UMP with the rotating speed under the UMP only as shown in Figure 10. As the rotating speed increases, the rotor orbit in Figure 9(a) (blue line) under NBB only changes from a group of semiellipses into a ring, which is obviously different from that under UMP only. The maximum amplitude variation under NBB only is in the form of a high-order curve, which has a phenomenal rise when rotating speeds changes from $1200 \mathrm{rpm}$ to $1800 \mathrm{rpm}$. It is noted that the effects of the unbalanced force gradually rise and then weaken the gravity effect relatively, which leads to the important rotor orbit changes. When both of the UMP and the NBB are taken into account, the rotor orbit at most rotating speeds represented as a ring, as shown in Figure 9(a) (red line). However, as rotating speed rises, the increasing UMP gradually limits the effect of the unbalanced force. The action of the gravity will stand out, resulting in the rotor orbit change at the high speed (2695 rpm). It can be found in Figure 9(b) that the maximum vibration amplitude variation with the increasing rotating speeds under the coupling effect of the UMP and NBB is in the form of a strongly nonlinear curve, and its amplitude is higher than that under the single action. The contribution of UMP under the combined action to amplitude is far more significant than that under UMP alone, which is consistent with the conclusion in [22]. The maximum amplitude under the coupling effect of the UMP and the NBB is not equal to the linear superposition of amplitudes under the single action. From Figure 10, it can also be found that when the rotating speed changes from $2000 \mathrm{rpm}$ to $2700 \mathrm{rpm}$, both the amplitude of the UMP and $\mathrm{NBB}$ under the combined effect has a decrease, which is different from those under single action. It is indicated that the accurate predicted results can be obtained only by considering the combined action, while the results under the single factor are distorted against the actual situation.

According to the proposed dynamic model, the frequency waterfall chart of dynamic response in $Y$ direction versus rotating speed under different cases is plotted in Figure 11. It can be found from Figure 11(a) that $\omega^{*}$ mainly appears in the frequency waterfall chart under UMP only, which rises nonlinearly. In Figure 11(b), the frequency spectrum under NBB only contains much more frequency components including $\omega^{*}, 2 \omega^{*}, \omega_{\mathrm{vc}}^{*}-\omega^{*}, 3 \omega^{*}, \omega_{\mathrm{vc}}^{*}, 4 \omega^{*}$, $\omega_{\mathrm{vc}}^{*}+\omega^{*}$, and $5 \omega^{*}$. The frequency components of $\left(\omega_{\mathrm{vc}}^{*} \pm n \omega^{*}, n=0,1\right)$ is closely related to the varying compliance frequency. However, as shown Figure 11(c), when the UMP is introduced into the analysis, the amplitude of $\omega_{\mathrm{vc}}^{*} \pm n \omega^{*}$ could become insignificant.

Figure 12 represents the amplitude variation of $\omega^{*}$ and $2 \omega^{*}$ versus rotating speed under different cases. Their amplitudes are listed and compared in Tables 5 and 6 , where $A_{U \& N}$ is assigned to the amplitude under both UMP and $\mathrm{NBB}$, and $A_{U}$ and $A_{N}$ denote the amplitude under UMP or NBB, respectively. In Figure 12, the amplitude curve of $\omega^{*}$ under the coupling effect of UMP and NBB has a relatively higher value than the one under NBB alone, which indicates that the introduction of UMP might aggravate the vibration of the motor rotor. We can see from Table 5 that $A_{U}$ is small for $\omega^{*}$ when the rotating speed is slow, but it has a remarkable impact on dynamic response of the rotor-bearing system. Hence, the shape of the rotor orbit under both of UMP and NBB is similar to the one under UMP alone as 

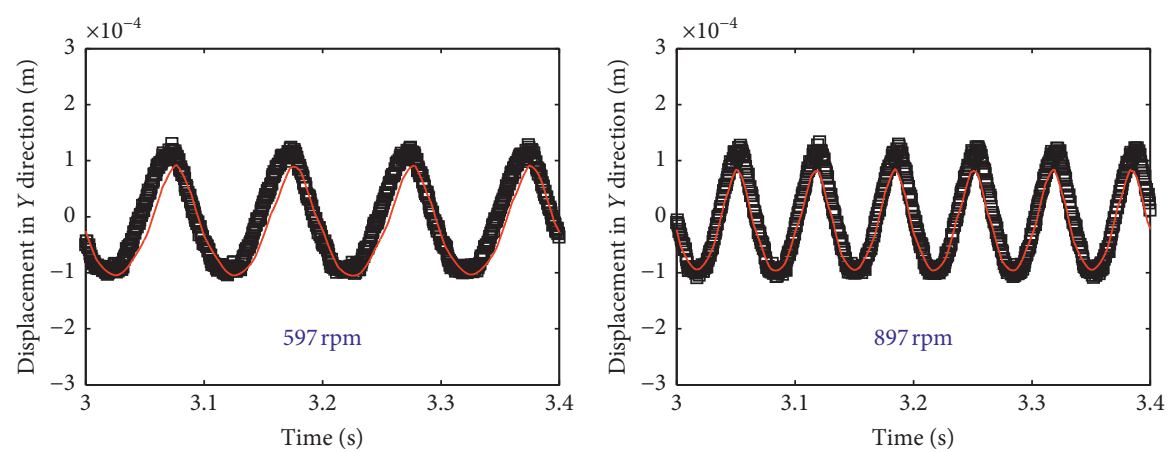

․ Experimental result

Experimental result

- Numerical result

- Numerical result
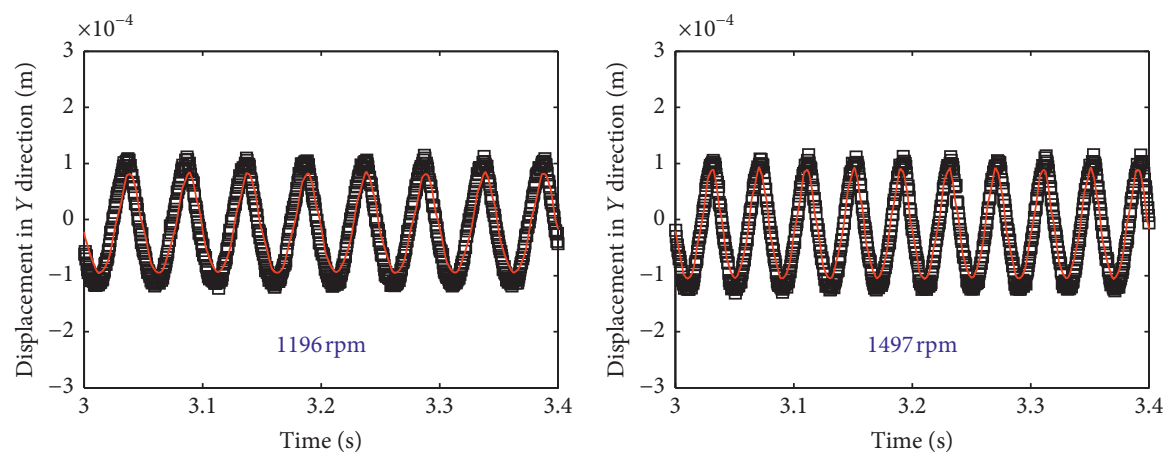

$\square \quad$ Experimental result

$\square \quad$ Experimental result

— Numerical result

_ Numerical result
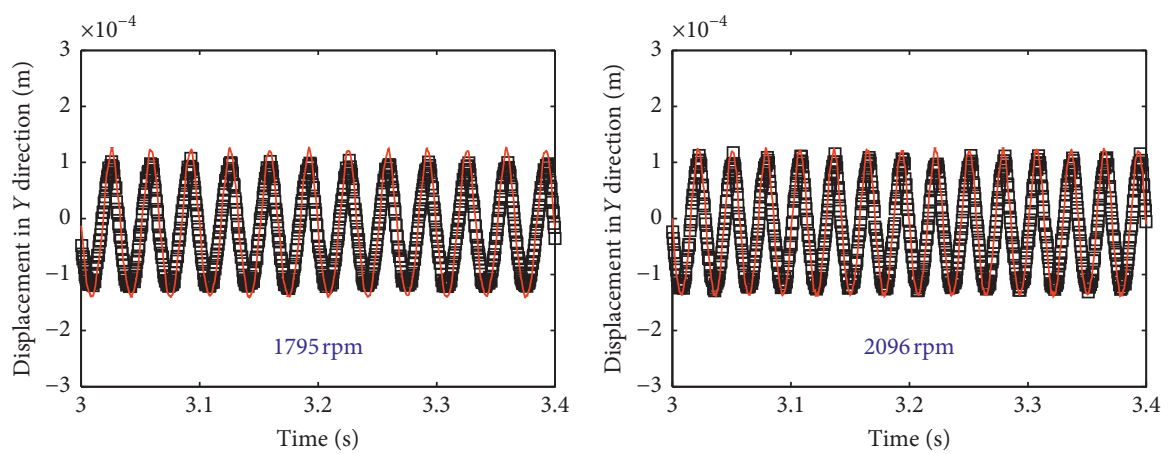

ㅁ Experimental result

Numerical result

Experimental result

- Numerical result
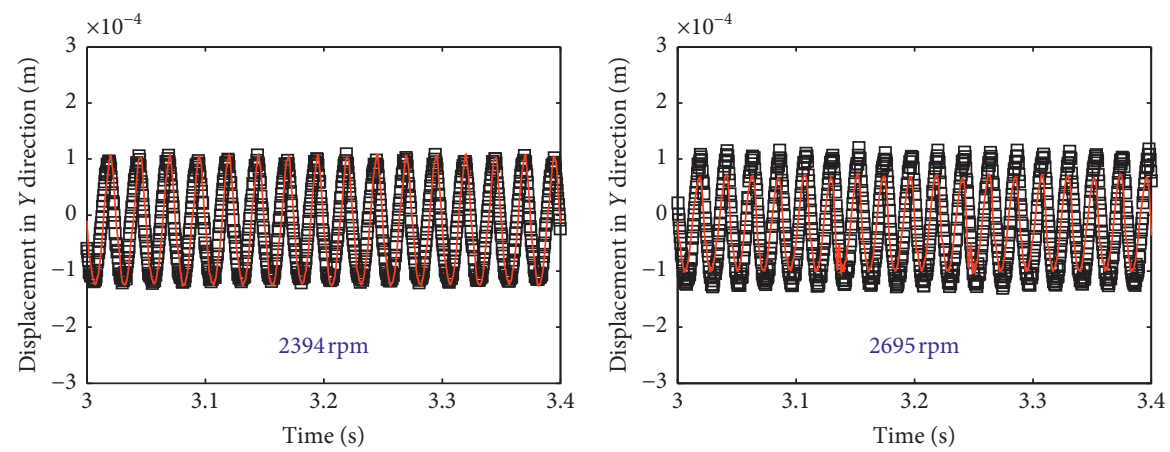

$\square \quad$ Experimental result

Experimental result

_ Numerical result

_ Numerical result

(a)

Figure 7: Continued. 

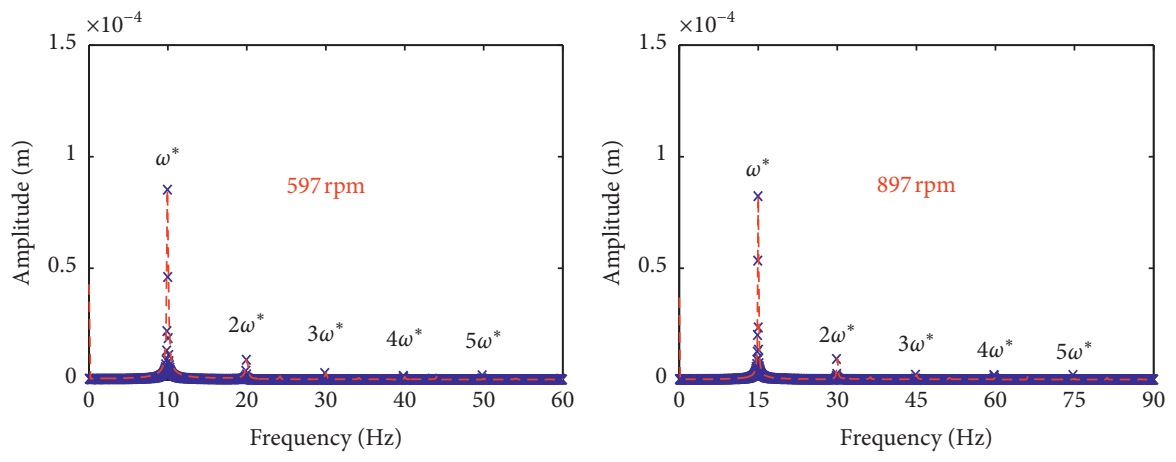

$\times \quad$ Experimental result

- - - Numerical result

$\times \quad$ Experimental result

- - - Numerical result
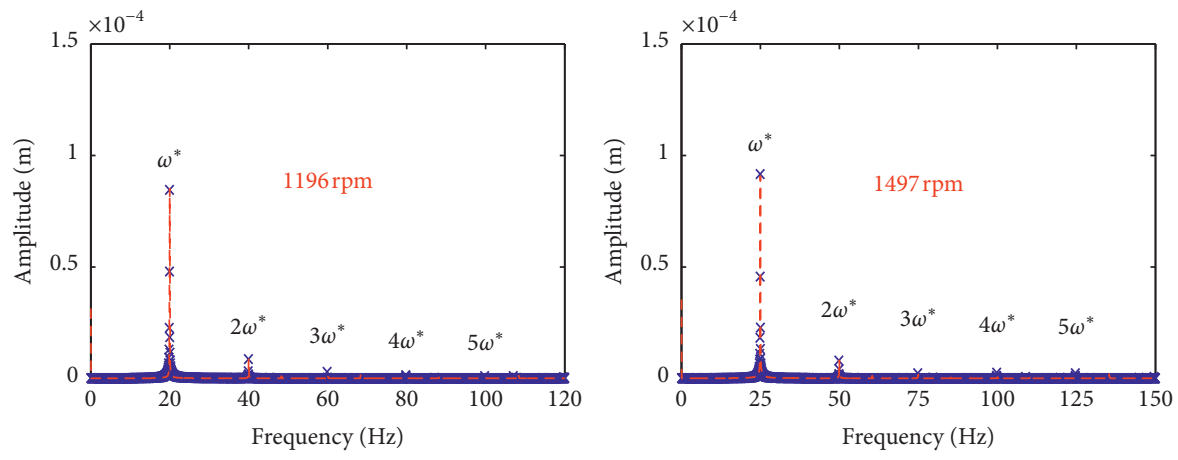

$\times \quad$ Experimental result

$\times \quad$ Experimental result

- . - Numerical result

- - Numerical result
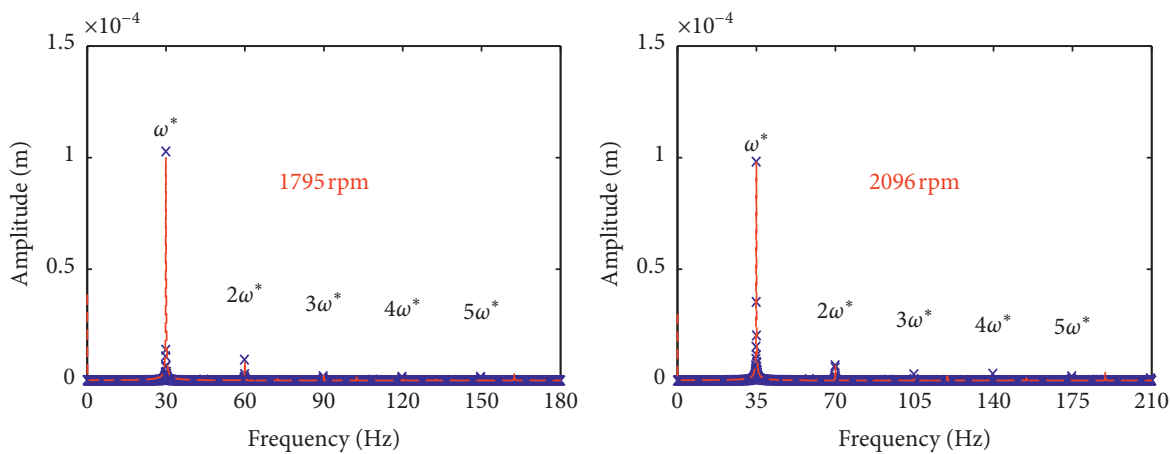

$\times \quad$ Experimental result

- _ - Numerical result

$\times \quad$ Experimental result

- - - Numerical result
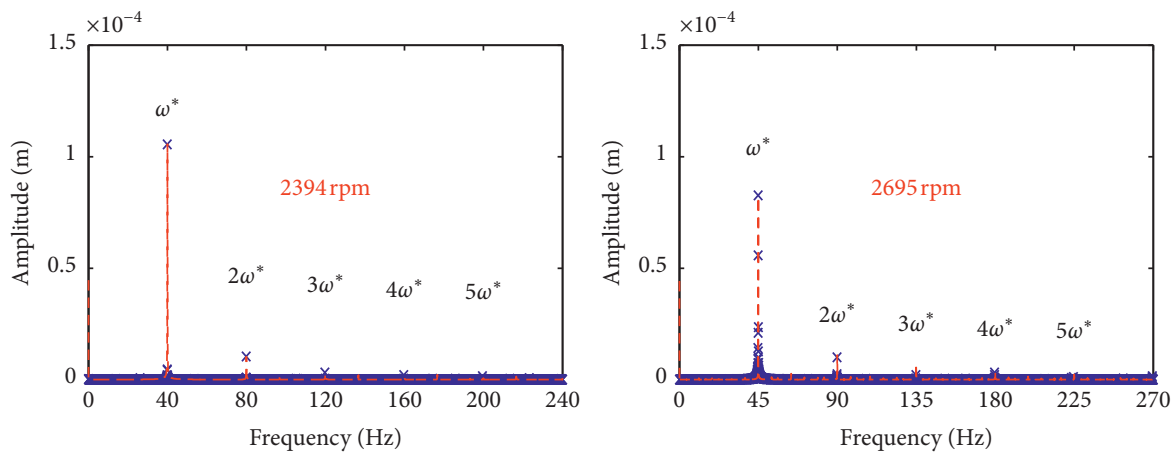

$\times$ Experimental result

$\times$ Experimental result

- - - Numerical result

- - - Numerical result

(b)

FIgURE 7: Comparison of experimental and numerical results of the motor rotor. (a) Dynamic response in $Y$ direction. (b) Frequency spectrum. 


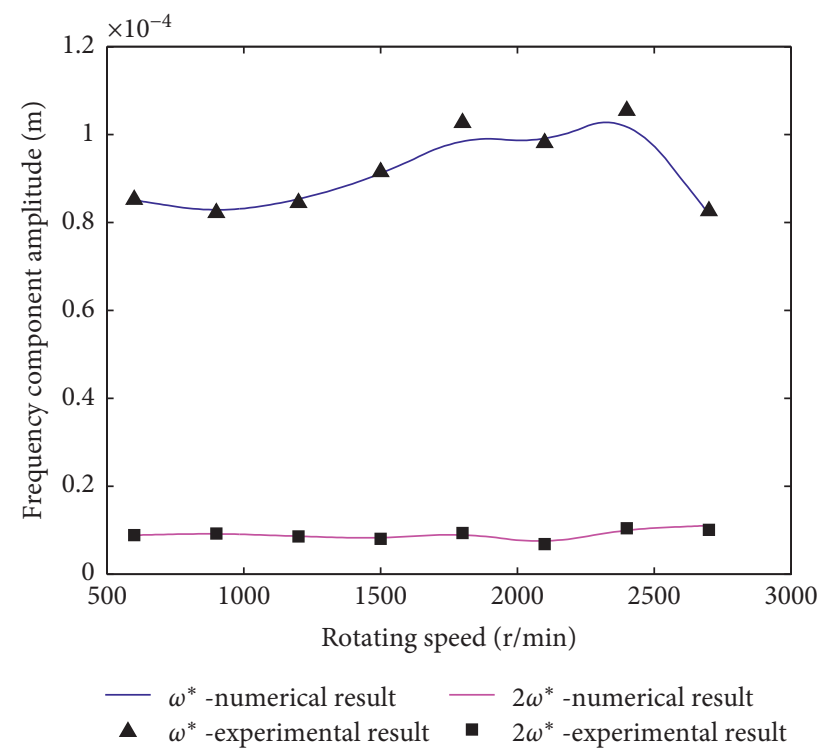

FIGURE 8: Amplitude variation of frequency components versus rotating speed.

TABLE 4: Rotating speed of the motor rotor versus supply frequency of power.

\begin{tabular}{lcccccccc}
\hline$\omega_{s}^{*}(\mathrm{~Hz})$ & 10 & 15 & 20 & 25 & 30 & 35 & 40 & 45 \\
$n_{r}(r / \mathrm{min})$ & 597 & 897 & 1196 & 1497 & 1795 & 2096 & 2394 & 2695 \\
\hline
\end{tabular}

Note. $\omega_{s}{ }^{*}$, supply frequency of power; $n_{r}$, rotating speed of the motor rotor.

shown in Figure 9(a). The strong nonlinearity of the amplitude variations of $\omega^{*}$ and $2 \omega^{*}$ under NBB only is dominated by the Hertzian contact force of the ball bearing, which plays an important part in the nonlinear behavior of the rotor of the induction motor. Once the UMP is taken into consideration, $A_{N}$ for $2 \omega^{*}$ in Table 6 at speed of $500 \mathrm{rpm} \sim 1300 \mathrm{rpm}$ will rise. Moreover, the coupling effect is not a simple sum because $A_{U \& N} \neq A_{U}+$ $A_{N}$ for both $\omega^{*}$ and $2 \omega^{*}$ in Tables 5-6. Thus, it can be concluded that the combine effect of the UMP and the $\mathrm{NBB}$ at room temperature on rotor vibration is nonlinear and important, whereas the results under the single factor are small and apparently cannot correspond to the actual situation.

4.2. The Influence of the Temperature. In our study, the thermal effect, which has a remarkable impact on the rotordynamic behaviors of motor, has been taken into consideration. As shown in Figure 13, when the rotating speed of the rotor is set to $1196 \mathrm{rpm}$, the amplitude variations of $\omega^{*}$ and $2 \omega^{*}$ versus temperature obtained through the numerical method are in good agreement with that of the test results. In Table 7 , it is noted that the difference between the predicted result and the experimental data is less than $3 \%$ and thus validates the presented multifield coupling dynamic model of the motor rotor system. It can also be found from Figure 13(a) that the amplitude curve of $\omega^{*}$ presents a nonlinear decreasing trend as the rotor surface temperature changing from $30^{\circ} \mathrm{C}$ to $50^{\circ} \mathrm{C}$. The reason is that when temperature of the iron core rises, the decrease of the air gap length might enhance the effect of the UMP. The UMP, which is in the opposite direction at this rotating speed, will then weaken the effect of the unbalanced force related to the mass eccentricity. However, due to the significant effect of the UMP on the ball bearing, the amplitude variation of $2 \omega^{*}$ is obviously different from that of $\omega^{*}$.

In Figure 14, the rotor orbit of all the three different cases represents little change versus temperature, which indicates that the shape of rotor orbits behaves insensitively to the increase of the temperature rise from $30^{\circ} \mathrm{C}$ to $50^{\circ} \mathrm{C}$. The corresponding amplitude of maximum vibration in $Y$ direction is given in Table 8 , where $V_{U \& N}$, denoting the amplitude of maximum vibration in $Y$ direction under both $\mathrm{UMP}$ and $\mathrm{NBB}$, is always higher than the others. $V_{U}$ as the maximum amplitude under UMP has a small rise, which is significantly different from the maximum vibration in $Y$ direction under NBB only. It is also found in Table 8 that the value of $V_{U \& N}$ is bigger than the sum of $V_{U}$ and $V_{N}$ at different temperatures. It is noted that the maximum vibration in $Y$ direction is closely associated with the amplitude of $\omega^{*}$ as given in Table 9, where $A_{U}$ for $\omega^{*}$ grows slowly and $A_{N}$ has a slight decrease.

Figure 15 shows the amplitude variation of $\omega^{*}$ and $2 \omega^{*}$ versus temperature under different cases, and their corresponding amplitude comparisons are given in Tables 9 and 10. From Figure 15(a), the amplitude curve of $\omega^{*}$ under the coupling effect of the magnetic field and ball bearing has a relatively higher value, but it decreases 

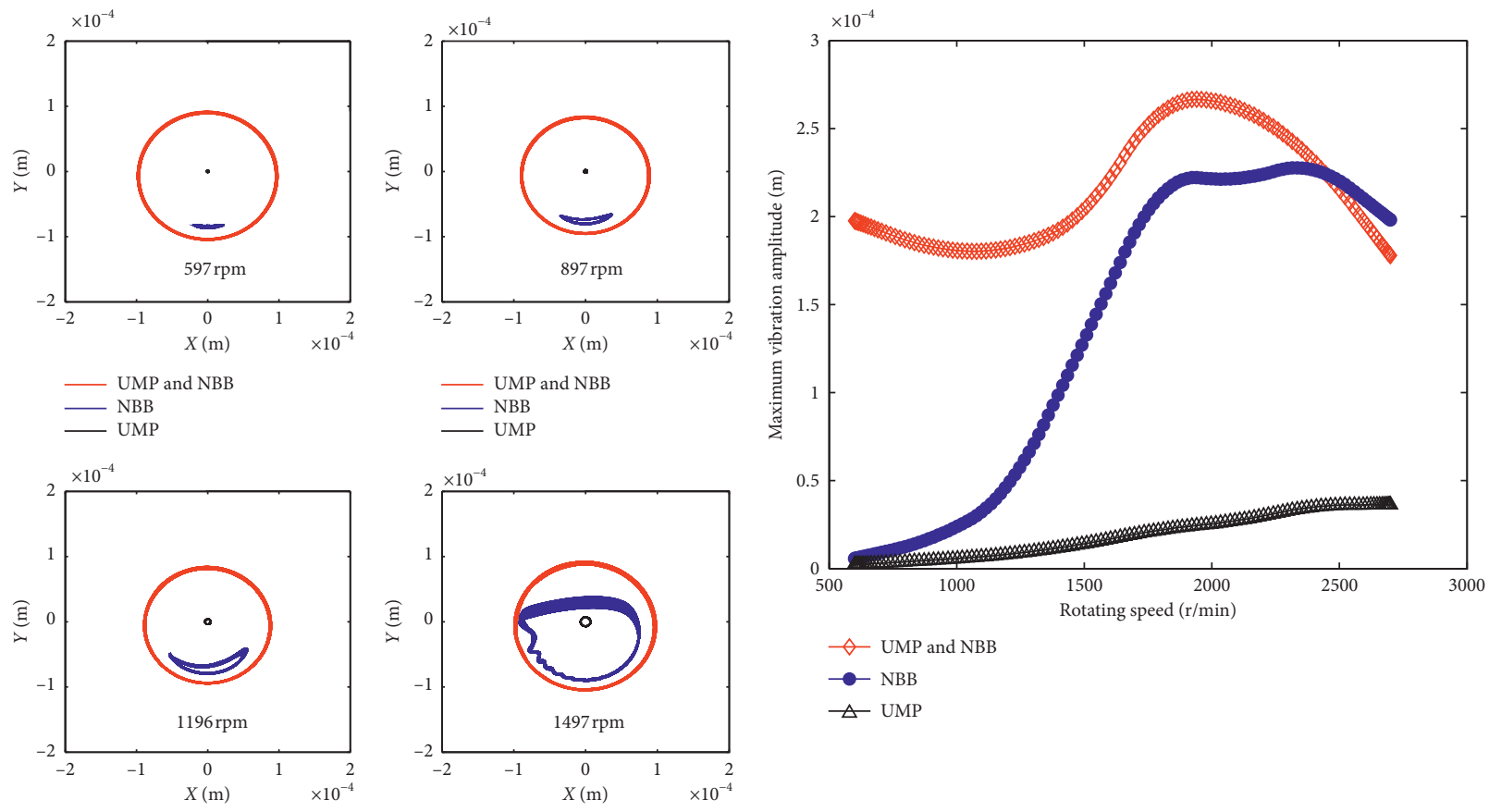

$\diamond$ UMP and NBB

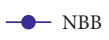

$\triangle$ UMP
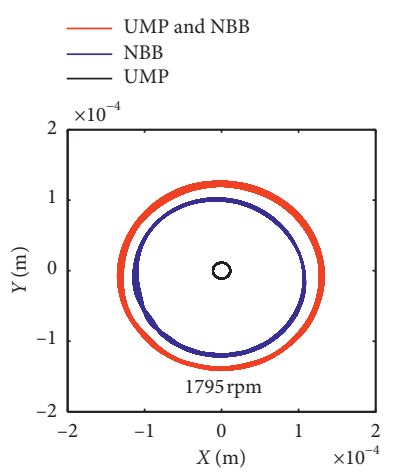

_ UMP and NBB

$$
\text { - UMP }
$$

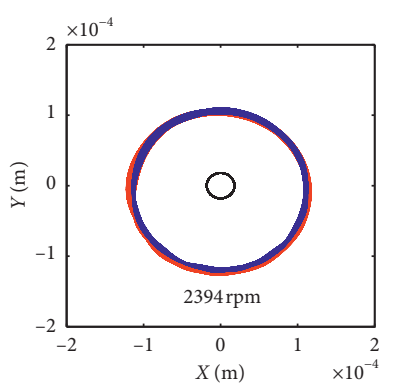

- UMP and NBB

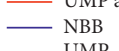

$$
\begin{aligned}
& \text { UMP and NBB } \\
& \text { NBB } \\
& \text { UMP }
\end{aligned}
$$

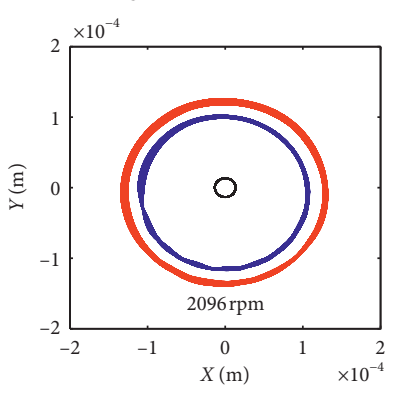

UMP and NBB
NBB

$$
\text { - UMP }
$$

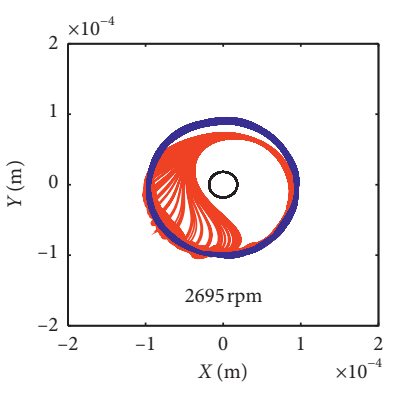

UMP and NBB

- NBB

(a)

(b)

FIGURE 9: Rotor dynamic response in $Y$ direction versus rotating speed under different cases. (a) Rotor orbit. (b) Maximum amplitude in $Y$ direction.

nonlinearly as the temperature rises. It shows that the interaction of the UMP and NBB makes the rotor dynamic behavior more sensitive to the change of the temperature. Under NBB alone, the frequency component of $2 \omega^{*}$ weakens with the increase of the temperature in Figure 15(b). As given in Table 10, although $A_{U}$ for $2 \omega^{*}$ is minor at different temperatures, the effect of UMP on the ball bearing-rotor system is significant and important, 


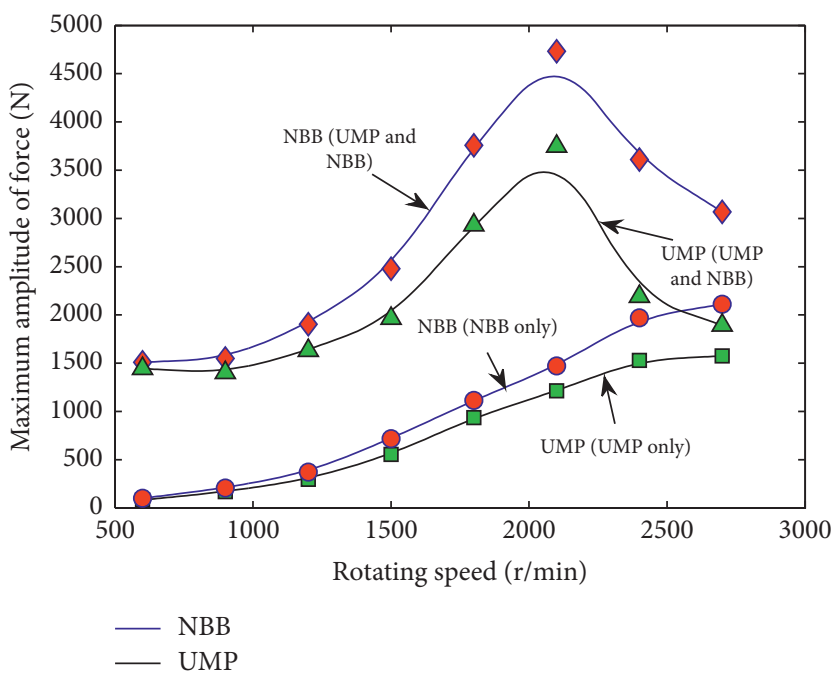

Figure 10: Maximum amplitude in $Y$ direction versus rotating speed under different cases.

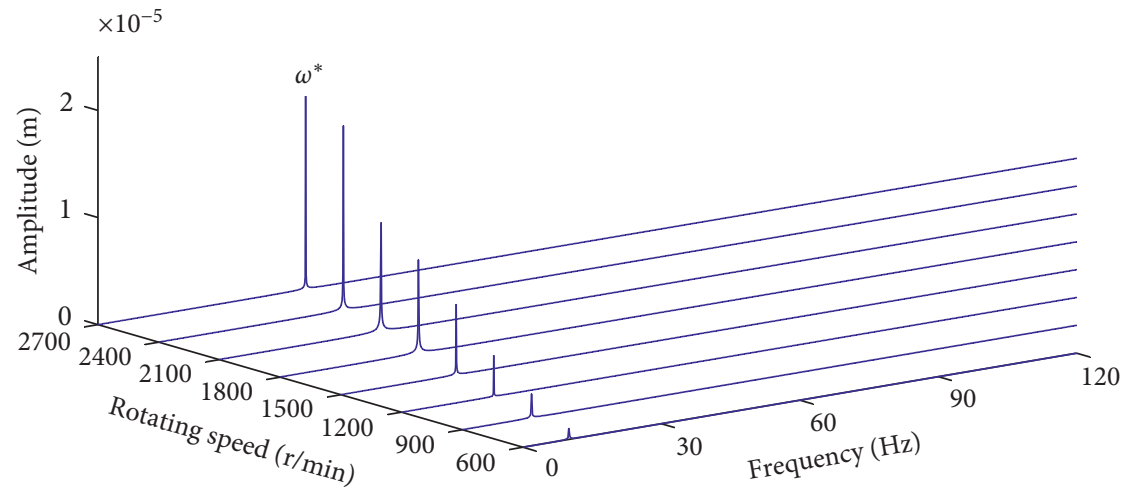

(a)

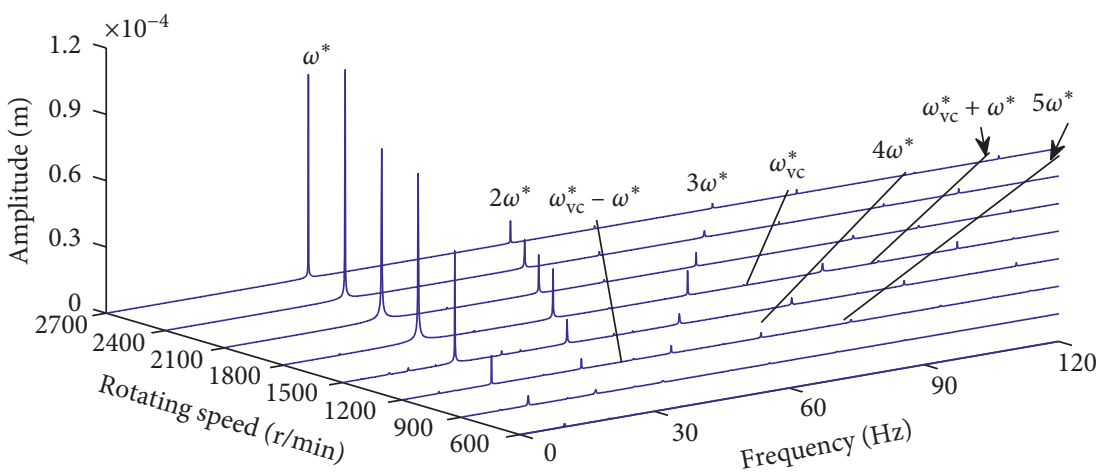

(b)

Figure 11: Continued. 


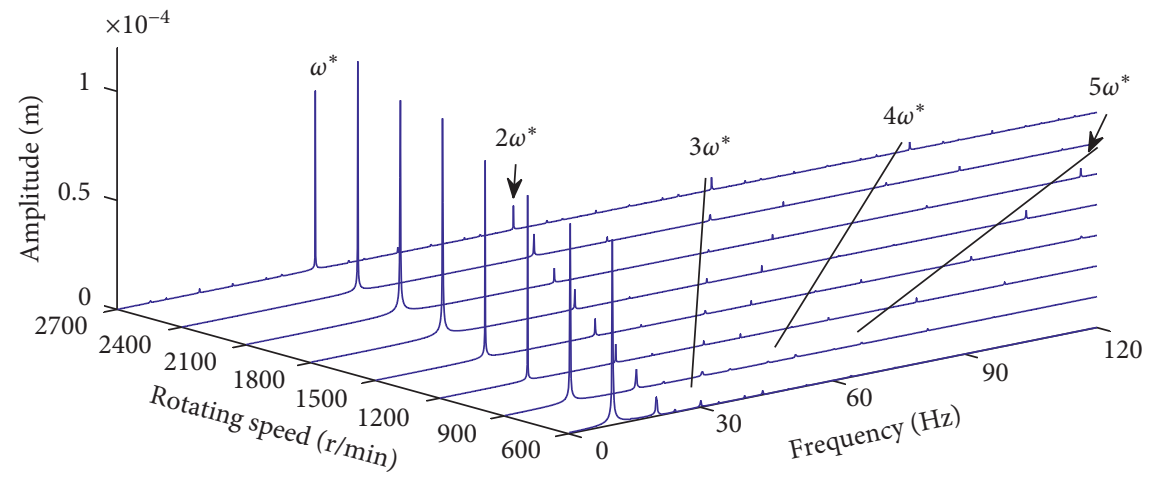

(c)

FIGURE 11: Frequency waterfall chart of dynamic response in $Y$ direction versus rotating speed under different cases. (a) UMP only. (b) NBB only. (c) UMP and NBB.

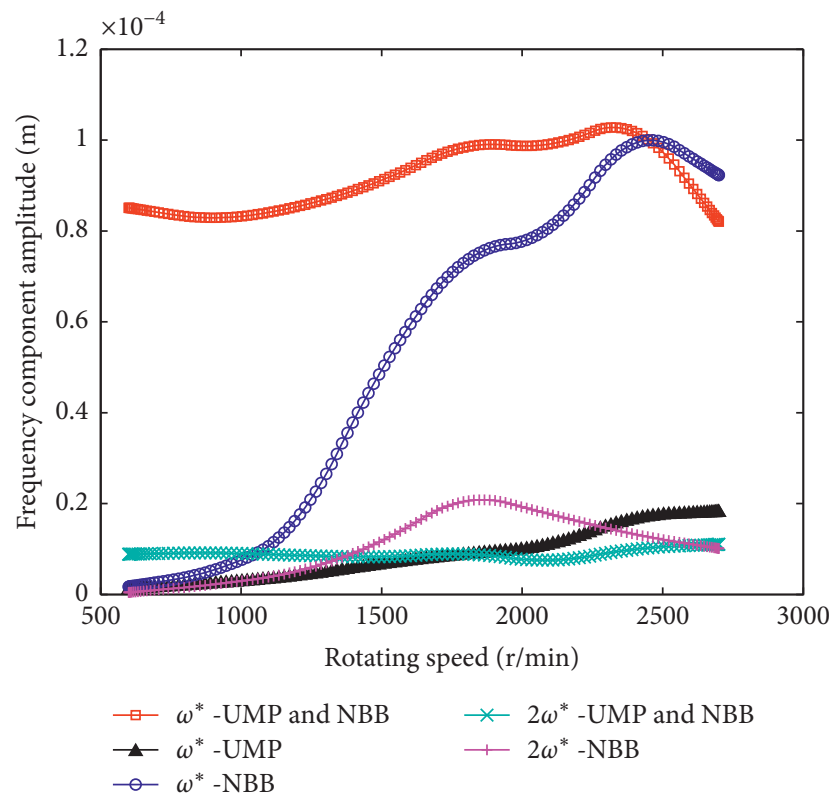

Figure 12: Amplitude variation of frequency components versus rotating speed under different cases.

TABLe 5: Amplitude comparison of frequency component $\omega^{*}$ under different cases.

\begin{tabular}{lccccc}
\hline Rotating speed $(\mathrm{rpm})$ & $A_{U \& N}(\mathrm{~m})$ & $A_{U}(\mathrm{~m})$ & $\left(A_{U} / A_{U \& N}\right) \times 100 \%(\%)$ & $A_{N}(\mathrm{~m})$ & $\left(A_{N} / A_{U \& N}\right) \times 100 \%(\%)$ \\
\hline 597 & $8.509 e-5$ & $1.076 e-6$ & 1.26 & $1.703 e-6$ & 2.00 \\
897 & $8.219 e-5$ & $2.25 e-6$ & 2.74 & $4.616 e-6$ & $1.325 e-5$ \\
1196 & $8.498 e-5$ & $3.846 e-6$ & 7.53 & $5.091 e-5$ & 15.59 \\
1497 & $9.076 e-5$ & $6.643 e-6$ & 8.72 & $7.635 e-5$ & 76.09 \\
1795 & $9.982 e-5$ & $8.771 e-6$ & 10.47 & $1.040 e-4$ & 79.49 \\
1795 & $9.794 e-5$ & $1.025 e-5$ & 16.35 & $1.040 e-4$ & 98.39 \\
2096 & $1.057 e-4$ & $1.728 e-5$ & 22.00 & $9.228 e-5$ & 112.52 \\
2695 & $8.201 e-5$ & $1.804 e-5$ & & \\
\hline
\end{tabular}

Note. $A_{U \& N}$, amplitude under both UMP and NBB; $A_{U}$, amplitude under UMP; $A_{N}$, amplitude under NBB.

which results in the massive changes to the amplitude curve of $2 \omega^{*}$ under NBB only. Thus, the accurate predicted dynamic response of the motor rotor versus temperature can be obtained only by considering the combine action. It can be concluded that the temperature rises.
According to the proposed multifield coupling dynamic model of the motor rotor system, the amplitude of $\omega^{*}$ and $2 \omega^{*}$ versus temperature at the rotating speed of $1795 \mathrm{rpm}$ and $2394 \mathrm{rpm}$ are investigated, and the correspond amplitude curves are shown in Figure 16. It can be found from 
TABLE 6: Amplitude comparison of frequency component $2 \omega^{*}$ under different cases.

\begin{tabular}{lccccc}
\hline Rotating speed $(\mathrm{rpm})$ & $A_{U \& N}(\mathrm{~m})$ & $A_{U}(\mathrm{~m})$ & $\left(A_{U} / A_{U \& N}\right) \times 100 \%$ & $A_{N}(\mathrm{~m})$ & $\left(A_{N} / A_{U \& N}\right) \times 100 \%(\%)$ \\
\hline 597 & $8.855 e-6$ & Minor & Minor & $4.328 e-7$ & 4.89 \\
897 & $9.273 e-6$ & Minor & Minor & $2.070 e-6$ & 22.32 \\
1196 & $8.581 e-6$ & Minor & Minor & $4.649 e-6$ & 54.18 \\
1497 & $8.081 e-6$ & Minor & Minor & $1.121 e-5$ & 138.72 \\
1795 & $9.336 e-6$ & Minor & Minor & $2.256 e-5$ & 241.65 \\
2096 & $6.815 e-6$ & Minor & Minor & $1.758 e-5$ & 257.96 \\
2394 & $1.026 e-5$ & Minor & Minor & $1.305 e-5$ & 121.19 \\
2695 & $1.102 e-5$ & Minor & Minor & $1.011 e-5$ & 51.74 \\
\hline
\end{tabular}

Note. $A_{U \& N}$, amplitude under both UMP and NBB; $A_{U}$, amplitude under UMP; $A_{U}$, amplitude under NBB.

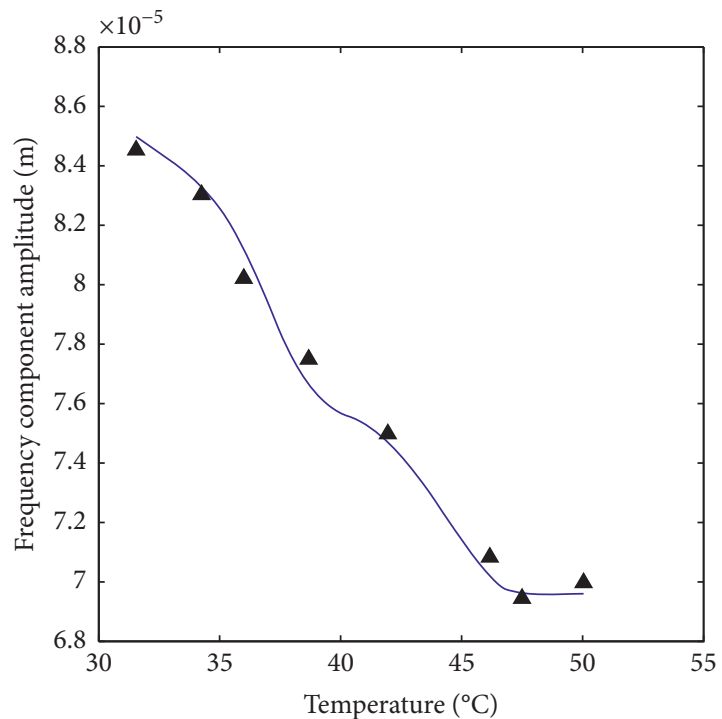

- $\omega^{*}$-numerical result

$\Delta \quad \omega^{*}$-experimental result

(a)

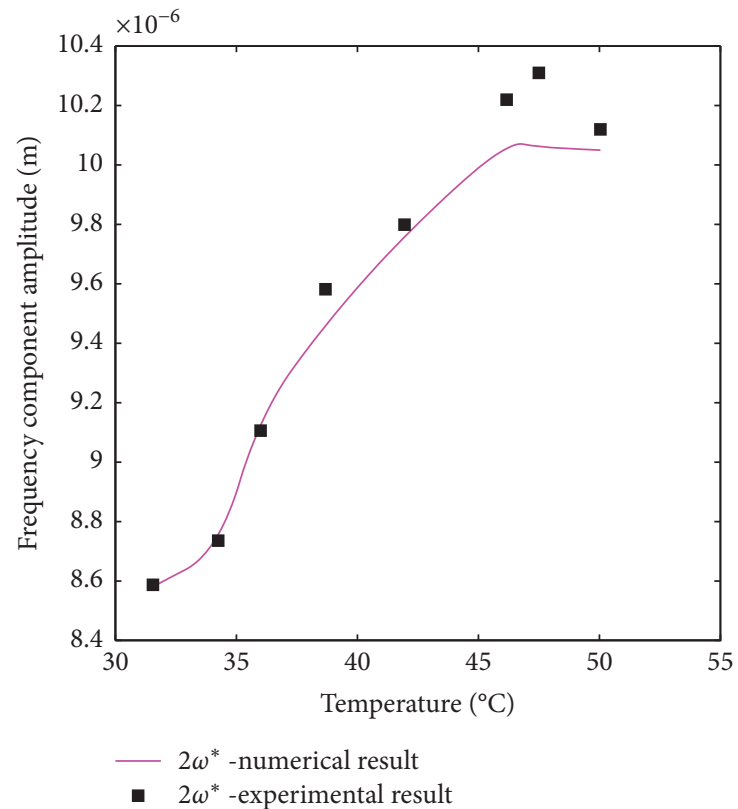

(b)

FIGURE 13: The comparison of the amplitude of frequency components versus temperature between numerical and experimental results: (a) $\omega^{*}$; (b) $2 \omega^{*}$.

TABLE 7: The comparison of the amplitude of frequency components versus temperature between numerical and experimental results at the rotating speed of $1196 \mathrm{rpm}$.

\begin{tabular}{lcccccccc}
\hline Temperature $\left({ }^{\circ} \mathrm{C}\right)$ & $31.55(\%)$ & $34.25(\%)$ & $36.00(\%)$ & $38.68(\%)$ & $41.95(\%)$ & $46.17(\%)$ & $47.50(\%)$ & $50.04(\%)$ \\
\hline$\omega^{*}$ & 0.53 & 0.51 & 1.50 & 1.91 & 0.21 & 1.26 & 0.17 & 0.53 \\
$2 \omega^{*}$ & 0.07 & 0.46 & 0.65 & 1.21 & 0.34 & 1.37 & 2.42 & 0.69 \\
\hline
\end{tabular}

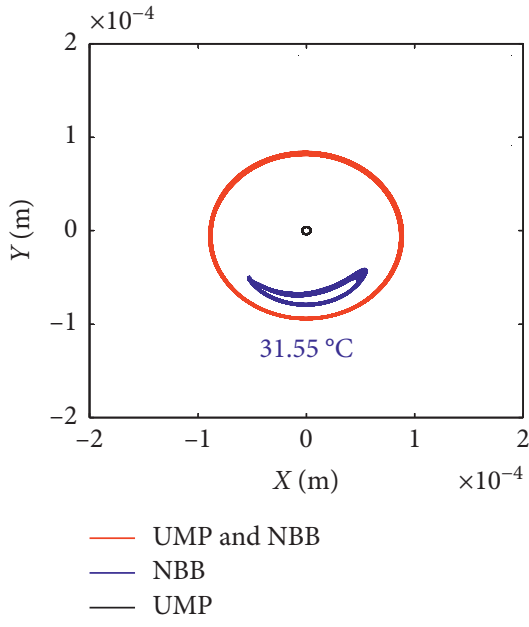

(a)

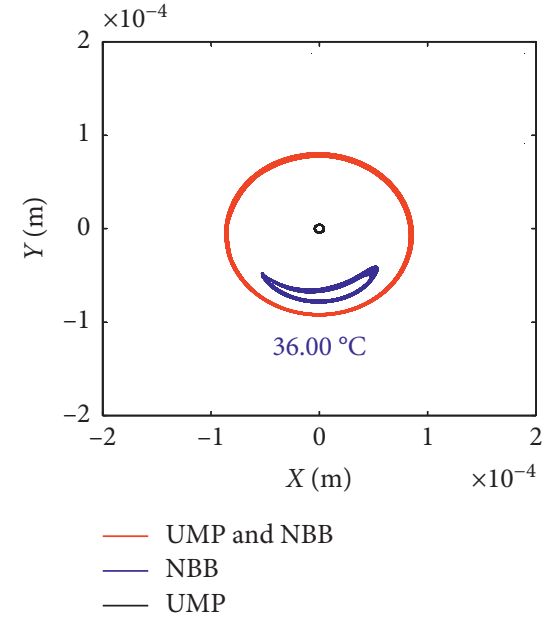

(b)

FIgure 14: Continued. 


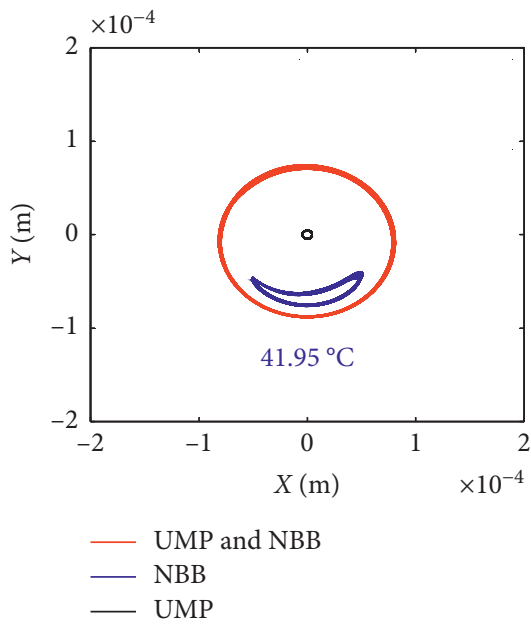

(c)

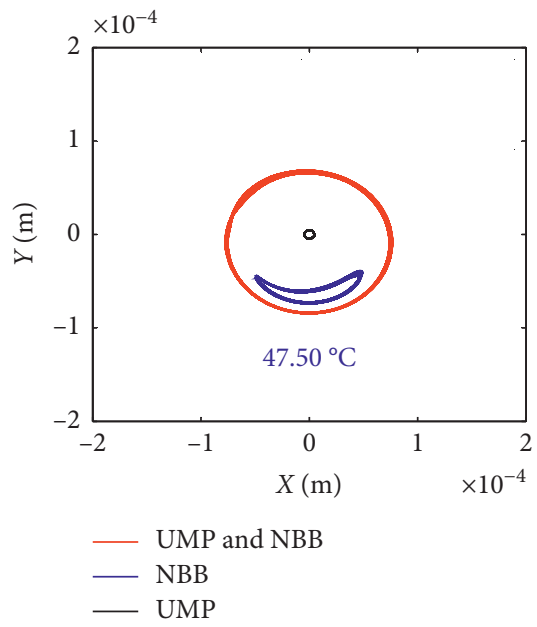

(d)

FigURE 14: Rotor orbit versus temperature under different cases.

TABLE 8: Amplitude of maximum vibration in $Y$ direction versus temperature under different cases.

\begin{tabular}{|c|c|c|c|c|c|}
\hline Temperature $\left({ }^{\circ} \mathrm{C}\right)$ & $V_{U \& N}(\mathrm{~m})$ & $V_{U}(\mathrm{~m})$ & $\left(V_{U} / V_{U \& N}\right) \times 100 \%(\%)$ & $V_{N}(\mathrm{~m})$ & $\left(V_{N} / V_{U \& N}\right) \times 100 \%(\%)$ \\
\hline 31.55 & $1.797 e-4$ & $7.772 e-6$ & 4.32 & $3.955 e-5$ & 22.00 \\
\hline 34.25 & $1.770 e-4$ & $7.894 e-6$ & 4.46 & $3.900 e-5$ & 22.04 \\
\hline 36.00 & $1.739 e-4$ & $8.381 e-6$ & 4.82 & $3.848 e-5$ & 22.13 \\
\hline 38.68 & $1.640 e-4$ & $8.446 e-6$ & 5.15 & $3.681 e-5$ & 22.44 \\
\hline 41.95 & $1.632 e-4$ & $8.914 e-6$ & 5.46 & $3.670 e-5$ & 22.48 \\
\hline 46.17 & $1.542 e-4$ & $9.106 e-6$ & 5.91 & $3.538 e-5$ & 22.95 \\
\hline 47.50 & $1.535 e-4$ & $9.106 e-6$ & 5.93 & $3.524 e-5$ & 22.96 \\
\hline 50.04 & $1.535 e-4$ & $9.105 e-6$ & 5.93 & $3.524 e-5$ & 22.96 \\
\hline
\end{tabular}

Note. $V_{U \& N}$, amplitude under both UMP and NBB; $V_{U}$, amplitude under UMP; $V_{N}$, amplitude under NBB.

TABle 9: Amplitude comparison of frequency component $\omega^{*}$ versus temperature under different cases.

\begin{tabular}{lccccc}
\hline Temperature $\left({ }^{\circ} \mathrm{C}\right)$ & $A_{U \& N}(\mathrm{~m})$ & $A_{U}(\mathrm{~m})$ & $\left(A_{N} / A_{U \& N}\right) \times 100 \%(\%)$ & $A_{N}(\mathrm{~m})$ & $\left(A_{N} / A_{U \& N}\right) \times 100 \%(\%)$ \\
\hline 31.55 & $8.498 e-5$ & $3.846 e-6$ & 4.53 & $1.325 e-5$ & 1.58 \\
34.25 & $8.345 e-5$ & $3.906 e-6$ & 5.09 & $1.321 e-5$ & $1.341 e-5$ \\
36.00 & $8.141 e-5$ & $4.147 e-6$ & 5.50 & $1.305 e-5$ & 15.83 \\
38.68 & $7.601 e-5$ & $4.179 e-6$ & 5.87 & $1.334 e-5$ & 17.17 \\
41.95 & $7.514 e-5$ & $4.411 e-6$ & 6.44 & $1.313 e-5$ & 17.75 \\
46.17 & $6.994 e-5$ & $4.506 e-6$ & 6.48 & $1.310 e-5$ & 18.77 \\
47.50 & $6.956 e-5$ & $4.506 e-6$ & 6.47 & $1.310 e-5$ & 18.83 \\
50.04 & $6.960 e-5$ & $4.505 e-6$ & 18.82 & \\
\hline
\end{tabular}

Note. $A_{U \& N}$, amplitude under both UMP and NBB; $A_{U}$, amplitude under UMP; $A_{N}$, amplitude under NBB.

Tables 11 and 12 that the difference between the predicted result and the experimental data is less than $6 \%$ at both speeds. It is shown in Figures 16(a) and 16(c) that both of the amplitude curves of $\omega^{*}$ have a nonlinear decreasing trend, which is similar to the variation of $\omega^{*}$ at the speed of $1196 \mathrm{rpm}$. However, the amplitude changes of $2 \omega^{*}$ at three 


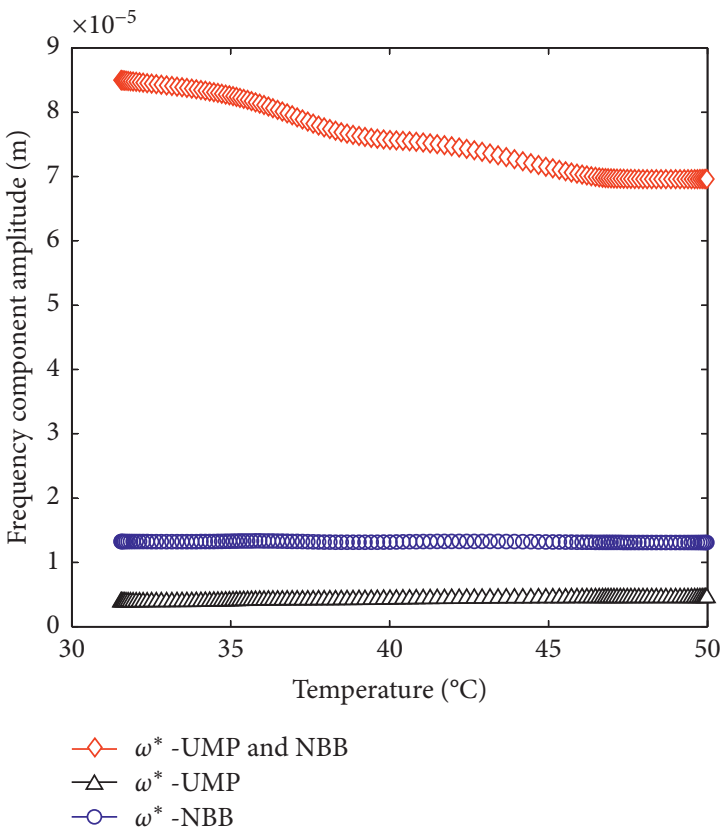

(a)

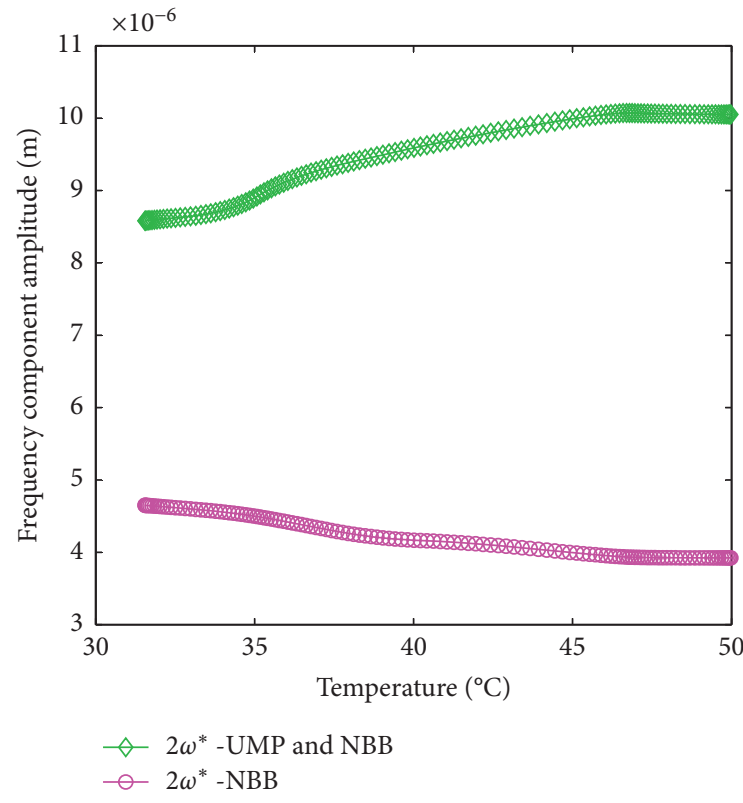

(b)

Figure 15: Amplitude variation of frequency components versus temperature under different cases.

TABLE 10: Amplitude comparison of frequency component $2 \omega^{*}$ versus temperature under different cases.

\begin{tabular}{lccccc}
\hline Temperature $\left({ }^{\circ} \mathrm{C}\right)$ & $A_{U \& N}(\mathrm{~m})$ & $A_{U}(\mathrm{~m})$ & $\left(A_{U} / A_{U \& N}\right) \times 100 \%$ & $A_{N}(\mathrm{~m})$ & $\left(A_{N} / A_{U \& N}\right) \times 100 \%(\%)$ \\
\hline 31.55 & $8.581 e-6$ & Minor & Minor & $4.649 e-6$ & 54.18 \\
34.25 & $8.696 e-6$ & Minor & Minor & $4.555 e-6$ & 52.38 \\
36.00 & $9.165 e-6$ & Minor & Minor & $4.425 e-6$ & 48.28 \\
38.68 & $9.466 e-6$ & Minor & Minor & $4.191 e-6$ & 44.27 \\
41.95 & $9.766 e-6$ & Minor & Minor & $4.129 e-6$ & 32.28 \\
46.17 & $1.008 e-5$ & Minor & Minor & $3.941 e-6$ & 39.10 \\
47.50 & $1.006 e-5$ & Minor & Minor & $3.923 e-6$ & 39.00 \\
50.04 & $1.005 e-5$ & Minor & Minor & $3.923 e-6$ & 39.03 \\
\hline
\end{tabular}

Note. $A_{U \& N}$, amplitude under both UMP and NBB; $A_{U}$, amplitude under UMP; $A_{N}$, amplitude under NBB.

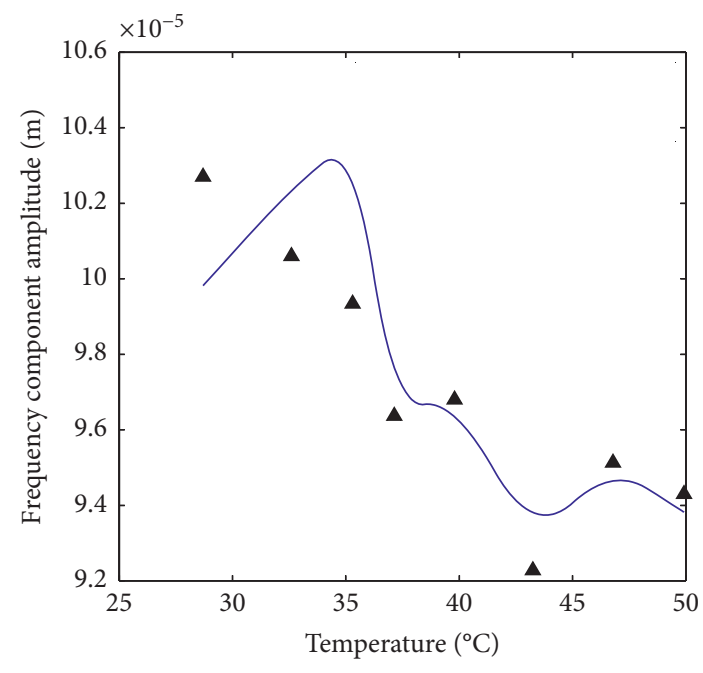

$\omega^{*}$-numerical result

$\Delta \quad \omega^{*}$-experimental result

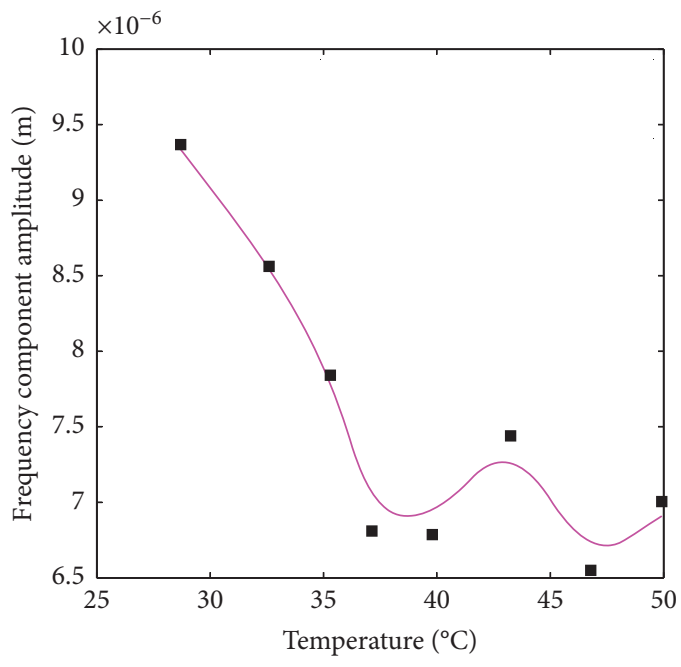

$2 \omega^{*}$-numerical result

- $2 \omega^{*}$-experimental result 


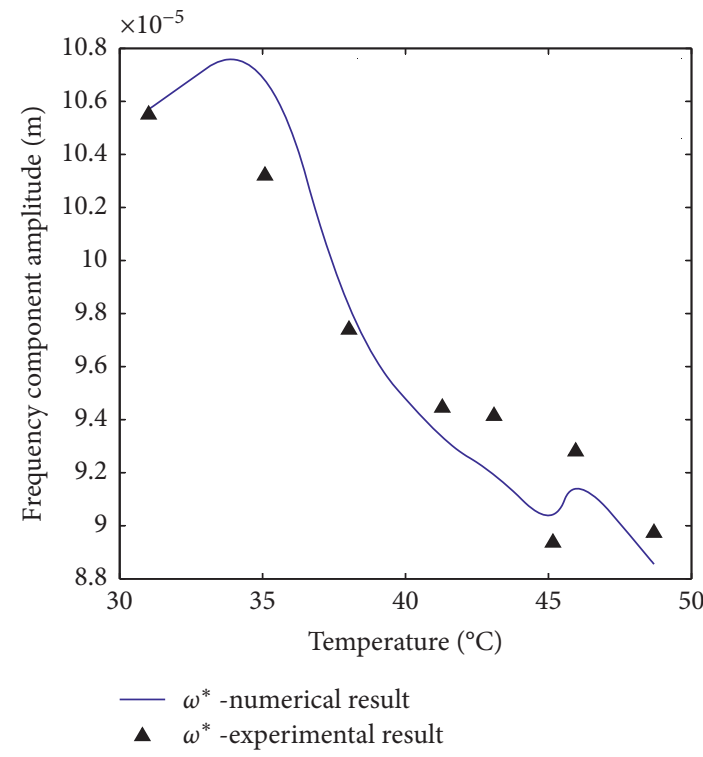

(c)

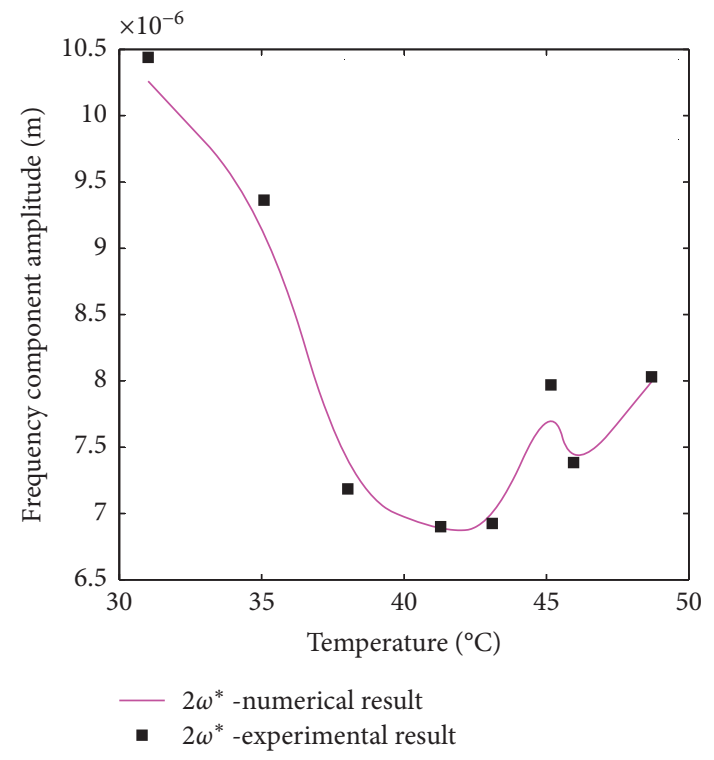

(d)

FIGURE 16: Amplitude variation of frequency components versus temperature at the different rotating speeds: (a) $1795 \mathrm{rpm}$; (b) $1795 \mathrm{rpm}$; (c) $2394 \mathrm{rpm}$; (d) $2394 \mathrm{rpm}$.

TABLE 11: The comparison of the amplitude of frequency components versus temperature between numerical and experimental results at the rotating speed of $1795 \mathrm{rpm}$.

\begin{tabular}{lcccccccc}
\hline Temperature $\left({ }^{\circ} \mathrm{C}\right)$ & $28.7(\%)$ & $32.6(\%)$ & $35.3(\%)$ & $37.13(\%)$ & $39.80(\%)$ & $43.25(\%)$ & $46.78(\%)$ & $49.92(\%)$ \\
\hline$\omega^{*}$ & 2.80 & 1.79 & 4.39 & 0.22 & 0.03 & 0.91 & 0.11 & 0.51 \\
$2 \omega^{*}$ & 0.34 & 0.12 & 0.03 & 1.86 & 1.47 & 0.16 & 0.81 & 1.37 \\
\hline
\end{tabular}

TABLE 12: The comparison of the amplitude of frequency components versus temperature between numerical and experimental results at the rotating speed of $2394 \mathrm{rpm}$.

\begin{tabular}{lcccccccc}
\hline Temperature $\left({ }^{\circ} \mathrm{C}\right)$ & $31.02(\%)$ & $35.08(\%)$ & $38.02(\%)$ & $41.29(\%)$ & $43.10(\%)$ & $45.16(\%)$ & $45.95(\%)$ & $48.69(\%)$ \\
\hline$\omega^{*}$ & 0.19 & 5.43 & 0.13 & 1.37 & 2.25 & 0.54 & 0.78 \\
$2 \omega^{*}$ & 1.72 & 0.45 & 0.39 & 0.67 & 0.40 & 1.09 & 1.46 & 0.47 \\
\hline
\end{tabular}

rotating speeds are different to each other, indicating that the temperature rises might have nonlinear impact on the motor rotor system supported by ball bearings.

\section{Conclusions}

In this study, a motor test platform controlled by a variable frequency converter is established, and the experiments under multiworking conditions are carried out for analyzing the rotordynamic characteristics of the motor system. A 2DOF magnetic-solid-thermal coupling dynamic model of the rotor-bearing system is presented, which includes the nonlinear effect of the ball bearing forces, the UMP, and the temperature rise of the motor rotor. The numerical results under different conditions are in good agreement with test data, thus validating the presented model. From the results presented in this study, the following conclusions can be drawn.
(1) In analysis of the rotor dynamic behaviors versus rotating speed, the dynamic response spectrum of the motor rotor contains the multiple frequency components $\left(\omega^{*}, 2 \omega^{*}, 3 \omega^{*}, 4 \omega^{*}\right.$, and $\left.5 \omega^{*}\right)$ generally. The rotor vibrations associated with $\omega^{*}$ and $2 \omega^{*}$ are more significant than those of other frequency components $\left(3 \omega^{*}, 4 \omega^{*}\right.$, and $\left.5 \omega^{*}\right)$.

(2) At low rotating speeds, UMP with slight amplitudes can have a remarkable effect on the dynamic characteristics of the system under the nonlinear effect of ball bearing forces. The influence of the UMP on the ball bearing-rotor system will restrict the amplitude of the frequency components related to the varying compliance frequency $\left(\omega_{\mathrm{vc}}^{*}-\omega^{*}, \omega_{\mathrm{vc}}^{*}\right.$, and $\left.\omega_{\mathrm{vc}}^{*}+\omega^{*}\right)$.

(3) Numerical and experimental results show that the effect of the unbalanced load gradually decreases with the increase of the temperature. The heat would decrease the vibration related to $\omega^{*}$ due to the 
temperature variation of the iron core and the ball bearing. Considering the thermal effect, the amplitude changes of $2 \omega^{*}$ at different rotating speeds are substantially different.

(4) The shape of the rotor orbit changes little with the increase of the temperature. But, the increase of temperature can lead to an important change of the amplitudes of $\omega^{*}$ and $2 \omega^{*}$. The contribution of temperature rise to the dynamic behavior of the rotor under the coupling effect of UMP and NBB is far more significant than that under the single action alone.

\section{Nomenclature}

a: $\quad$ The semimajor axis of projected contact ellipse

$A_{C F}$ : The contact area of insulation layer and iron core

$A_{C 1}$ : The area of heat dissipation of the windings

$A_{F i}$ : The area of heat dissipation surface of corresponding parts

$b$ : $\quad$ The semiminor axis of projected contact ellipse

$B(\theta, t)$ : The magnetic flux density of air gap magnetic field

$c$ : The damping coefficient of the motor

$c_{i}, c_{o}$ : The internal and outer radial clearance between the inner, outer race, and rolling elements

$d_{m}$ : The pitch diameter of the ball bearing

$D_{b}, D_{b 0}$ : The diameters of the bearing balls under the specific temperature and room temperature

$D_{p}: \quad$ The bearing pitch diameter

$e: \quad$ The distance of mass eccentricity

$f_{0}$ : A factor depending upon type of bearing and method of lubrication

$f_{l}$ : $\quad$ A factor depending upon bearing design and relative bearing load

$F_{\beta}$ : $\quad$ A coefficient depends on the magnitude and direction of the applied load

$F(\theta, t)$ : The fundamental MMF of the air gap

$F^{B}$ : The nonlinear ball bearings forces

$F^{E}$ : The unbalanced magnetic pull

$F_{j}: \quad$ The amplitude of the fundamental MMF of the excitation current of the rotor

$h_{v}$ : The film coefficient of heat transfer

$H$ : $\quad$ The bearing frictional power loss

$H_{A}$ : The total heat generated by the ball bearing

$k_{f}$ : The thermal conductivity

$k_{i j}, k_{o j}$ : The load-deflection constants between the inner and outer ball races

K: $\quad$ The contact stiffness of ball bearing

$K_{j}: \quad$ The contact stiffness of bearing

$l_{E}$ : The length of the windings ends

$L$ : $\quad$ The length of rotor core

$n$ : $\quad$ The rotating speed of rotor

$n_{b}$ : The rotating speed of the ball bearing

$n_{s}$ : The spinning speed of the bearing balls normal to the contact area

$N$ : The number of rolling elements

$m: \quad$ The rotor mass

$M: \quad$ The torque applied on the ball bearings

$M_{v}$ : The viscous friction torque
$M_{l}: \quad$ The torque due to applied load

$M_{s}$ : $\quad$ The torque due to the spinning force

$M_{g y}$ : The torque due to the gyroscopic force acting on the bearing balls

$p: \quad$ The pole pair number of the motor

$p_{f}, p_{C}$ : The power losses of the iron core and copper windings

Q: $\quad$ The load for each bearing ball

$Q_{j}: \quad$ The contact force of the

$j^{\text {th }}$ : Rolling element

Re: $\quad$ The Reynolds number for a rotating ball

$R_{1}$ : The outer radius of rotor

$R_{10}$ : The rotor outer radius under the normal condition of room temperature

$R_{C 1}$ : The thermal resistance from the end of the windings to the air

$R_{C 2}$ : The thermal resistance from the surface of the windings near the duct to the air

$R_{C F}$ : The thermal resistance between the copper windings and iron core

$R_{F 1}$ : The thermal resistance from the surface of the iron core near the duct to the air

$R_{F 2}$ : The thermal resistance from the inner surface of the iron core to the air

$R_{F 3}$ : The thermal resistance from the outer surface of the iron core to the air

$R_{\mathrm{Cu}}$ : The total thermal resistance of rotor windings

$R_{\mathrm{Fe}}$ : The total thermal resistance of iron core

$S: \quad$ The area of surface

$S_{1}$ : $\quad$ The external area of a bearing housing

$S_{2}$ : $\quad$ The total area of the surface of the bearing balls

$t: \quad$ Time

$u_{s}$ : The surface circumference of the conductor together with the insulating layer

$V_{r}$ : The relative velocity between the sliding velocity of the bearing ball and the velocity of the compressed air

$W: \quad$ The gravity of rotor

$W_{h}, D_{h}$ : The geometry sizes of bearing housing

$x, y: \quad$ The co-ordinates of the rotor center

$Z$ : $\quad$ The number of the rotor slots

$\Lambda(\theta, t)$ : The magnetic permeance per unit of the air gap

$\alpha: \quad$ The contact angle of ball bearing

$\alpha_{C 1}$ : The heat transfer coefficient of the windings ends

$\beta: \quad$ The ball attitude angle

$\gamma_{j}$ : The coefficient about the relationship of contact deformation and ball bearing force

$\delta_{0}$ : The mean air gap distance

$\delta_{C 1}$ : The thickness of the insulation layer of copper windings ends

$\delta_{C F}$ : The total thickness of insulation between copper windings and iron core

$\delta_{e}: \quad$ The transient displacement of rotor center

$\delta_{F i}$ : The thickness of the insulation layer

$\delta_{j}$ : The contact deformation of the $j^{\text {th }}$ rolling element

$\varepsilon: \quad$ The relative eccentricity $\varepsilon=\delta_{e} / \delta_{0}$

$\varepsilon_{m o}: \quad$ The complete elliptic integral of the second kind with modulus $\left[1-(b / a)^{2}\right]^{1 / 2}$ 
$\theta: \quad$ The value of angular coordinate

$\mu_{0}: \quad$ The air permeance

$\sigma: \quad$ The Maxwell stress

$\varphi_{j}: \quad$ The angular location of $j^{\text {th }}$ rolling element

$\varphi_{0}$ : The initial angular location of the rolling element

$\mu$ : $\quad$ The coefficient of friction

$v_{0}$ : The dynamic viscosity of lubricant

$v_{\text {air }}$ : The kinematic viscosity of the air

$\omega, \omega^{*}$ : The rotational speed of motor rotor, the angular velocity $\omega$ and $\omega^{*}=\omega /(2 \pi)$

$\omega_{c}, \omega_{c}{ }^{*}$ : The rotating speed of the cage, the angular velocity $\omega_{c}$ and $\omega_{c}^{*}=\omega_{c} /(2 \pi)$

$\omega_{s}, \omega_{s}^{*}$ : The angular velocity and frequency of the power, $\omega_{s}^{*}=\omega_{s} /(2 \pi)$

$\omega_{v c}$, The angular velocity and frequency of the ball

$\omega_{v c}{ }^{*}: \quad$ passage vibration, $\omega_{v c}^{*}=\omega_{v c} /(2 \pi)$

$\omega_{R}$ : The angular velocity of the bearing ball about its own axis

$\omega_{m}: \quad$ The orbital angular velocity of the bearing ball

$\lambda_{C F}$ : The thermal conductivity

$\lambda_{C 1}$ : The thermal conductivity of the windings ends

$\lambda_{F i}: \quad$ The thermal conductivity of the insulation layer

$\bar{\lambda}_{1}$ : Thermal expansion coefficient of the rotor core

$\bar{\lambda}_{2}$ : The thermal expand coefficient of the bearing balls

$\Delta T_{C}, \quad$ The temperature rise of the copper windings and

$\Delta T_{F}: \quad$ the rotor iron core.

\section{Appendix}

\section{The Calculation of the Temperature Rise Constants}

The thermal resistance between the copper windings and iron core can be expressed as

$$
R_{C F}=\frac{\delta_{C F}}{\lambda_{C F} A_{C F}},
$$

where $\delta_{C F}$ is the total thickness of insulation between copper windings and iron core, $\lambda_{C F}$ is the thermal conductivity, and $A_{C F}$ is the contact area of insulation layer and iron core.

The thermal resistance between copper windings ends and air can be calculated by

$$
R_{C 1}=\left(\frac{\delta_{C 1}}{\lambda_{C 1} A_{C 1}}+\frac{1}{\alpha_{C 1} A_{C 1}}\right),
$$

where $\delta_{C 1}$ is the thickness of the insulation layer of copper windings ends, $\lambda_{C 1}$ and $\alpha_{C 1}$ are the thermal conductivity and the heat transfer coefficient of the windings ends, respectively, $A_{C 1}$ is the area of heat dissipation of the windings, which is given as $A_{C 1}=l_{E} u_{s} Z, l_{E}$ is the length of the windings ends, $u_{s}$ is the surface circumference of the conductor together with the insulating layer, and $Z$ is the number of the rotor slots.

The calculation method of the thermal resistance between the windings and the air in a radial duct $\left(R_{\mathrm{C} 2}\right)$ is similar to that of the thermal resistance at the windings ends
$\left(R_{C 1}\right)$. The expression of $\left(R_{C 2}\right)$ is the same as equation (A.2) except that the corresponding parameter is different in values.

The surface heat resistance of radial air ducts and inner and outer surfaces to air can be calculated by the following formula:

$$
R_{F i}=\frac{\delta_{F i}}{\lambda_{F i} A_{F i}}, \quad i=1,2,3,
$$

where $\lambda_{F i}, \delta_{F i}$, and $A_{F i}$ are the thermal conductivity, the thickness of the insulation layer, and the area of heat dissipation surface of corresponding parts, respectively.

The viscous friction torque for ball bearings operating at moderate speed and under proper load can be expressed as follows:

$$
\begin{cases}M_{v}=10^{-7} \cdot f_{0} \cdot\left(v_{0} \cdot n\right)^{2 / 3} \cdot d_{m}^{3}, & v_{0} \cdot n \geq 2000 \\ M_{v}=160 \times 10^{-7} \cdot f_{0} \cdot d_{m}^{3}, & v_{0} \cdot n \leq 2000\end{cases}
$$

where $v_{0}$ is the dynamic viscosity of lubricant, and $f_{0}$ is a factor depending upon type of bearing and method of lubrication. The friction torque due to applied load can also be determined empirically by the following equation:

$$
M_{l}=f_{l} F_{\beta} d_{m}
$$

where $f_{l}$ is a factor depending upon bearing design and relative bearing load, $F_{\beta}$ depends on the magnitude and direction of the applied load, and $d_{m}$ is the pitch diameter of the ball bearing.

If the motion of the a raceway relative to the ball was merely a spinning about the normal to the center of the contact area and all other relative surface velocities are being reduced to zero, the magnitude of the spinning moment is given as

$$
M_{s}=3\left(\frac{\mu \mathrm{Q} a \varepsilon}{8}\right)
$$

where $\mu$ is the coefficient of friction, $Q$ is the load for each bearing ball, and $\varepsilon$ is the complete elliptic integral of the second kind with modulus $\left[1-(b / a)^{2}\right]^{1 / 2} \cdot a$ and $b$ are the semimajor axis and semiminor axis of projected contact ellipse, respectively.

The gyroscopic torque at each bearing ball location can be described as follows:

$$
M_{g y}=\left(\frac{1}{60}\right) \cdot \rho \cdot \pi \cdot D_{b}^{5} \cdot \omega_{R} \cdot \omega_{m} \cdot \sin \beta,
$$

where $\omega_{R}$ is the angular velocity of the bearing ball about its own axis, $\omega_{m}$ is the orbital angular velocity of the bearing ball, and $\beta$ is the ball attitude angle.

Finally, taking the four types of the torque acting on the bearing balls into consideration, the total heat generated by the ball bearing can be determined as

$$
\begin{aligned}
H_{A} & =1.047 \times 10^{-4} \cdot \sum n_{j} \cdot M_{j} \\
& =1.047 \times 10^{-4} \times\left(n_{b}\left(M_{v}+M_{1}+M_{g y}\right)+n_{s} \cdot M_{s}\right),
\end{aligned}
$$


where $n_{b}$ is the rotating speed of the ball bearing, and $n_{s}$ is the spinning speed of the bearing balls normal to the contact area.

In quiescent air, heat transfer by convection from the housing external surface may be approximated by using an outside film coefficient as follows:

$$
h_{v 1}=2.3 \times 10^{-5} \times \Delta T^{0.25} .
$$

The external area of a bearing housing or pillow block can be approximate as

$$
S_{1}=\pi D_{h}\left(W_{h}+0.5 D_{h}\right),
$$

where $W_{h}$ and $D_{h}$ are the geometry sizes of bearing housing. Therefore, the heat transfer between the surface of the ball bearing and the air can be expressed as follows:

$$
H_{1}=h_{v 1} \cdot S_{1} \cdot \Delta T \text {. }
$$

At the same time, the heat convection between the bearing balls and the compressed air flow is a significant way to take out the heat generated by the ball bearing. The coefficient of heat transfer for air flow past a sphere is given as [14]

$$
h_{v 2}=\left[2+\left(0.25+3 \times 10^{-4} \operatorname{Re}^{1.6}\right)^{0.5}\right] \frac{k_{f}}{D_{b}}, \quad 100<\operatorname{Re}<3 \times 10^{5} \text {, }
$$

where $D_{b}$ is the diameter of the ball, $k_{f}$ is the thermal conductivity, and Re is the Reynolds number for a rotating ball, which can be expressed as

$$
\operatorname{Re}=\frac{D_{b 0} \cdot V_{r}}{v_{\text {air }}}
$$

where $v_{\text {air }}$ is the kinematic viscosity of the air, and $V_{r}$ is the relative velocity between the sliding velocity of the bearing ball and the velocity of the compressed air.

Thus, the heat transfer between the ball bearing and the compressed air flow is obtained:

$$
H_{2}=h_{v 2} \cdot S_{2} \cdot \Delta T,
$$

where $S_{2}$ is the total area of the surface of the bearing balls.

\section{Data Availability}

The data used to support the findings of this study are included within the article and publicly available.

\section{Conflicts of Interest}

The authors declare that they have no conflicts of interest.

\section{Acknowledgments}

The authors are very grateful to Pengkai Jia for the help in experiment.

\section{References}

[1] D. G. Dorrell, "Calculation of unbalanced magnetic pull in small cage induction motors with skewed rotors and dynamic rotor eccentricity," IEEE Transactions on Energy Conversion, vol. 11, no. 3, pp. 483-488, 1996.

[2] M. Bradford, "Unbalanced magnetic pull in a 6-pole induction motor," Proceedings of the Institution of Electrical Engineers, vol. 115, no. 11, pp. 1619-1627, 1968.

[3] R. Belmans, A. Vandenput, and W. Geysen, "Calculation of the flux density and the unbalanced pull in two pole induction machines," Archiv für Elektrotechnik, vol. 70, no. 3, pp. 151-161, 1987.

[4] D. Guo, F. Chu, and D. Chen, "The unbalanced magnetic pull and its effects on vibration in a three-phase generator with eccentric rotor," Journal of Sound and Vibration, vol. 254, no. 2, pp. 297-312, 2002.

[5] A. Khalili, S. Boroujeni, and N. Takorabet, "Dynamic simulation of unbalanced magnetic force in doubly-fed induction machine with inter-turn short-circuited stator," Iranian Journal of Science and Technology, vol. 8, no. 2, pp. 1-9, 2020.

[6] H. Kim, J. Nerg, T. Choudhury, and J. T. Sopanen, "Rotordynamic simulation method of induction motors including the effects of unbalanced magnetic pull," IEEE Access, vol. 8, no. 4 , pp. 21631-21643, 2020.

[7] C. T. Walters, "The dynamics of ball bearings," Journal of Lubrication Technology, vol. 93, no. 1, pp. 1-10, 1971.

[8] P. K. Gupta, "Dynamics of rolling-element bearings-part IV: ball bearing results," Journal of Lubrication Technology, vol. 101, no. 3, pp. 319-326, 1979.

[9] S. Saito, "Calculation of nonlinear unbalance response of horizontal jeffcott rotors supported by ball bearings with radial clearances," Journal of Vibration and Acoustics, vol. 107, no. 4, pp. 416-420, 1985.

[10] C. Bai and Q. Xu, "Dynamic model of ball bearings with internal clearance and waviness," Journal of Sound and Vibration, vol. 294, no. 1, pp. 23-48, 2006.

[11] A. Rafsanjani and S. Abbasion, "Nonlinear dynamic modeling of surface defects in rolling element bearing systems," Journal of Sound and Vibration, vol. 319, no. 3, pp. 1150-1174, 2009.

[12] S. Jafarari and R. Rohani, "Experimental and numerical study of an angular contact ball bearing vibration response with spall defect on the outer race," Archive of Applied Mechanics, vol. 90 , no. 2, pp. 2487-2511, 2020.

[13] T. Harris, Rolling Bearing Analysis, Wiley, Hoboken, NY, USA, 2nd edition, 1984.

[14] Y.-R. Jeng and P.-Y. Huang, "Predictions of temperature rise for ball bearings," Tribology Transactions, vol. 46, no. 1, pp. 49-56, 2003.

[15] J. Schmied, "Hot spots in turboexpander bearings: case history, stability analysis, measurements and operational experience," in Proceedings of the ASME Turbo Expo 2008: Power for Land, Sea and Air, Berlin, Germany, June 2008.

[16] J. Lorenz and B. Murphy, "Case study of morton effect shaft differential heating in a variable-speed rotating electric machine," in Proceedings of the ASME Turbo Expo 2011, Vancouver, Canada, June 2011.

[17] D. Panara, L. Baldassarre, and D. Griffini, Numerical Prediction and Experimental Validation of Rotor thermal Instability, Turbomachinery \& Pump Symposia, Houston, TX, USA, 2015.

[18] G. Aydin, Y. Sinan, and B. S. Zihni, “An integrated model for performance optimization of aerospace bearings considering 
high-speed rotations and temperature variations," Jounal of the American Helicopter Society, vol. 63, no. 1, pp. 12-40, 2018.

[19] T. Plantegenet, M. Arghir, M.-A. Hassini, and P. Jolly, "The thermal unbalance effect induced by a journal bearing in rigid and flexible rotors: experimental analysis," Tribology Transactions, vol. 63, no. 1, pp. 52-67, 2020.

[20] H. Im, H. H. Yoo, and J. Chung, "Dynamic analysis of a BLDC motor with mechanical and electromagnetic interaction due to air gap variation," Journal of Sound and Vibration, vol. 330, no. 8, pp. 1680-1691, 2011.

[21] H. Chuan and L. Gan, "Investigation of the power losses in induction machines with rotor eccentricity," Electrical Engineering, vol. 102, no. 3, pp. 1393-1403, 2020.

[22] S. Guo and C. Bai, "Coupling effect of unbalanced magnetic pull and ball bearing on nonlinear vibration of motor rotor system," Journal of Vibration and Control, Article ID 0981320, 2020.

[23] S. Chen, Design of the Motor, China Machine Press, Beijing, China, 2nd edition, 2000.

[24] T. Harris, Rolling Bearing Analysis, Wiley, Hoboken, NY, USA, 4th edition, 2001.

[25] S. Champman, Electric Machinery Fundamentals, McGrawHill Press, New York, NY, USA, 4th edition, 2012. 